\title{
Identification of potential genes in upper tract urothelial carcinoma using next-generation sequencing with bioinformatics and in vitro analyses
}

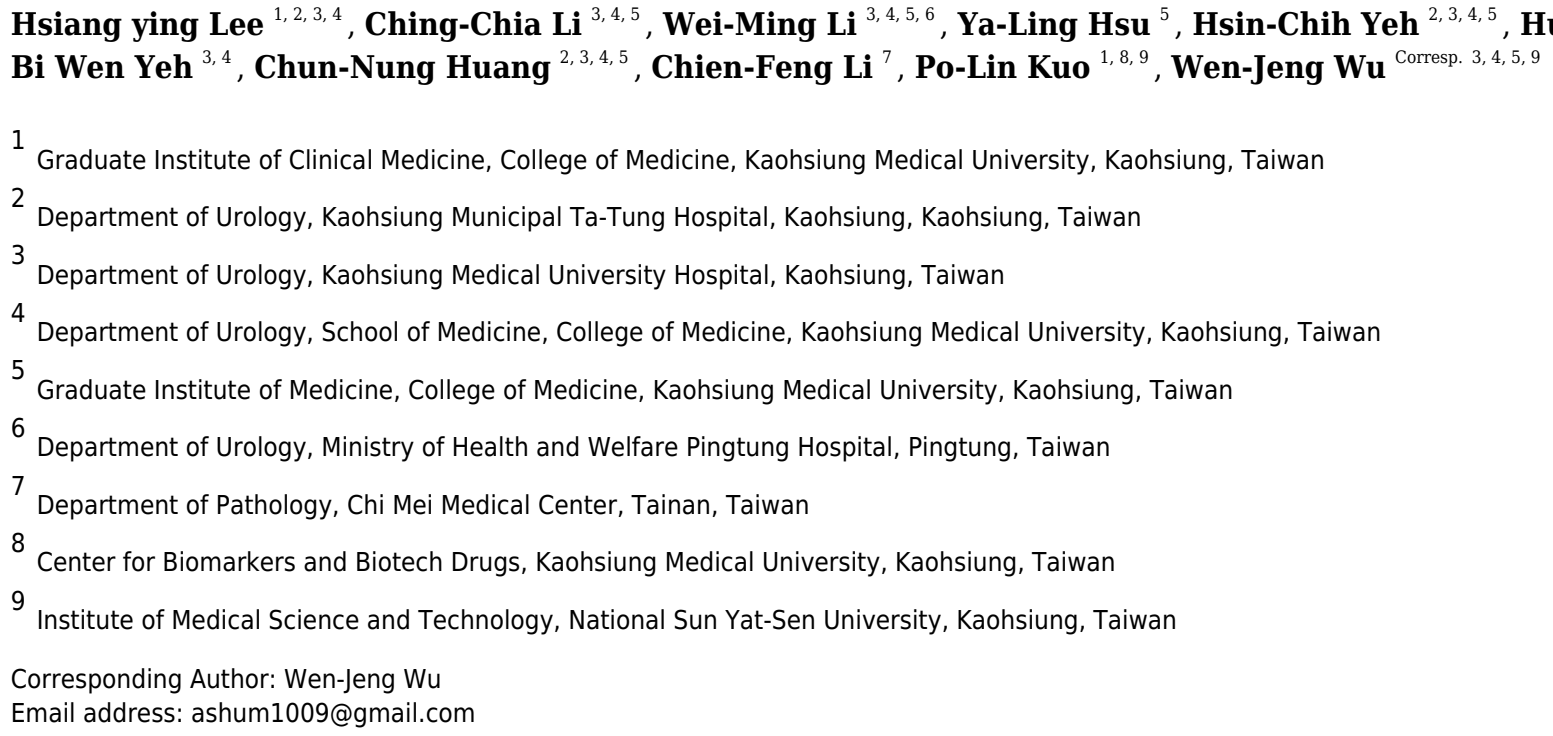

Background We aimed to identify prognostic biomarkers of upper tract urothelial carcinomas (UTUCs), including microRNAs (miRNAs) and genes which account for only $5 \%$ to $10 \%$ of all urothelial carcinomas (UCS). In Taiwan, this figure is markedly higher, where it can reach up to $30 \%$ of UC cases. Materials and Methods Using next-generation sequencing (NGS), we analyzed two pairs of renal pelvis tumors and adjacent normal urothelial tissues to screen miRNAs and messenger RNAs. By combining bioinformatics analysis from miRmap, Gene Expression Omnibus (GEO), and Oncomine and Ingenuity ${ }^{\circledR}$ Pathway Analysis databases, we identified candidate genes. To search for upstream miRNAs with exact target binding sites, we used miRmap, TargetScan, and miRDB to enforce evidence. Then, we clarified gene and protein expression through an in vitro study using western blot analysis and quantitative real-time reverse transcriptase-PCR. Results Interactions between selected target genes obtained using the NGS and miRmap methods were assessed through a Venn diagram analysis. Six potential genes, namely, PDE5A, RECK, ZEB2, NCALD, PLCXD3 and CYBRD1 showed significant differences. Further analysis of gene expression from the GEO dataset indicated lower expression of PDE5A, RECK, ZEB2, and CYBRD1 in bladder cancer tissue than in normal bladder mucosa, which indicated that PDE5A, RECK, ZEB2, and CYBRD1 may act as tumor suppressors in UTUC. In addition, we compared the expression of these genes in various UC cell lines (RT4, 
BFTC905, J82, T24, UMUC3, 5637, BFTC 909, UMUC14) and found decreased expression of PDE5A in muscle-invasive UC cells compared with the RT4 cell line. Furthermore, lower PDE5A expression in tumor specimens was also demonstrated in paired UTUC tissues (normal and tumor) from 20 patients. Conclusions Our findings suggest these candidate genes may play crucial roles in UTUC progression. We propose that these markers may be potential targets both as prognostic factors and for therapeutic strategies as clarified by in vitro and in vivo experiments. PDE5A also potentially presents tumor suppressor genes, as identified by comparing the expression between normal and tumor specimens. 
1 Identification of potential genes in upper tract urothelial carcinoma using next-generation

2

3

4

5

6 Hsiang-Ying Lee MD, MS ${ }^{1,2,3,4}$, Ching-Chia Li MD, PhD ${ }^{3,4,5}$, Wei-Ming Li MD, PhD $3,4,5,6$, Ya-

7 Ling Hsu $\mathrm{PhD}^{5}$, Hsin-Chih Yeh MD, PhD $2,3,4,5$, Hung-Lung Ke MD, PhD ${ }^{3,4,5}$, Bi-Wen Yeh,

$8 \mathrm{PhD}^{3,4}$, Chun-Nung Huang MD, PhD ${ }^{2,3,4,5}$, Chien-Feng Li MD, PhD 7 , Po-Lin Kuo PhD ${ }^{1,8,9}$,

9 Wen-Jeng $\mathrm{Wu} \mathrm{MD,} \mathrm{PhD} \mathrm{3,4,5,9*}$

$10{ }^{1}$ Graduate Institute of Clinical Medicine, College of Medicine, Kaohsiung Medical University,

11 Kaohsiung, Taiwan

12 2Department of Urology, Kaohsiung Municipal Ta-Tung Hospital, Kaohsiung, Taiwan

$13{ }^{3}$ Department of Urology, Kaohsiung Medical University Hospital, Kaohsiung, Taiwan

$14{ }^{4}$ Department of Urology, School of Medicine, College of Medicine, Kaohsiung Medical

15 University, Kaohsiung, Taiwan

$16{ }^{5}$ Graduate Institute of Medicine, College of Medicine, Kaohsiung Medical University,

17 Kaohsiung, Taiwan

18

${ }^{6}$ Department of Urology, Ministry of Health and Welfare Pingtung Hospital, Pingtung, Taiwan

${ }^{7}$ Department of Pathology, Chi Mei Medical Center, Tainan, Taiwan

20 
$21{ }^{9}$ Institute of Medical Science and Technology, National Sun Yat-Sen University, Kaohsiung,

22 Taiwan

23 * Corresponding author: Wen-Jeng Wu, M.D., PHD. Email: ashum1009@gmail.com

24

25

26

27

28

29

30

31

32

33

34

35

36

37

\section{Background}

We aimed to identify prognostic biomarkers of upper tract urothelial carcinomas (UTUCs), including microRNAs (miRNAs) and genes which account for only $5 \%$ to $10 \%$ of all urothelial carcinomas (UCs). In Taiwan, this figure is markedly higher, where it can reach up to $30 \%$ of UC cases.

\section{Materials and Methods}

Using next-generation sequencing (NGS), we analyzed two pairs of renal pelvis tumors and adjacent normal urothelial tissues to screen miRNAs and messenger RNAs. By combining bioinformatics analysis from miRmap, Gene Expression Omnibus (GEO), and Oncomine and Ingenuity ${ }^{\circledR}$ Pathway Analysis databases, we identified candidate genes. To search for upstream miRNAs with exact target binding sites, we used miRmap, TargetScan, and miRDB to enforce evidence. Then, we clarified gene and protein expression through an in vitro study using western blot analysis and quantitative real-time reverse transcriptase-PCR.

\section{Results}

Interactions between selected target genes obtained using the NGS and miRmap methods were assessed through a Venn diagram analysis. Six potential genes, namely, PDE5A, RECK, ZEB2, NCALD, PLCXD3 and CYBRD1 showed significant differences. Further analysis of gene expression from the GEO dataset indicated lower expression of PDE5A, RECK, ZEB2, and CYBRD1 in bladder cancer tissue than in normal bladder mucosa, which indicated that PDE5A, RECK, ZEB2, and CYBRD1 may act as tumor suppressors in UTUC. In addition, we compared the expression of these genes in various UC cell lines (RT4, BFTC905, J82, T24, UMUC3, 5637, BFTC 909, UMUC14) and found decreased expression of PDE5A in muscle-invasive UC cells compared with the RT4 cell line. Furthermore, by using paired UTUC and normal tissues from 20 patients, lower PDE5A expression was also demonstrated in tumor specimens.

\section{Conclusions}

Our findings suggest these candidate genes may play some roles in UTUC progression. We propose that these markers may be potential targets clarified by in vitro and in vivo experiments. PDE5A also potentially presents tumor suppressor genes, as identified by comparing the

Peer) reviewing PDF | (2020:10:54841:2:0:NEW 27 Mar 2021) 
54 55

56

57

58

59

60

61

62

63

64

65

66

67

68

69

70

71

72

73

expression between normal and tumor specimens.

Keywords: upper tract urothelial carcinoma, next-generation sequencing, bioinformatics, target genes, PDE5A

\section{Introduction}

Urothelial carcinomas (UCs) are derived from the urothelium and divided into upper and lower tract UCs according to tumor location. Upper tract urothelial carcinoma (UTUC), originating from the renal pelvis and ureter presents unique features in Taiwan compared to those in the Western regions. UTUC accounts for only 5\%-10\% of all UCs in Western countries but up to approximately $30 \%$ of UCs in Taiwan [1]. In addition, according to a recent report from the Taiwan Cancer Registry in 2013, the age-standardized incidence per 100,000 person-years was $4.09 \%$ and $4.37 \%$ in men and women, respectively, with a male-to-female ratio of 1:1.2 [2]. Conversely, Shariat et al. calculated that the incidence in men is twice that in women [3]. Specific environmental risk factors in Taiwan have been cited, including black foot disease and Chinese herb nephropathy $[4,5]$. However, although herbs with aristolochic acid components have been prohibited and environmental contamination by arsenic has improved, the incidence of UTUC has not decreased.

Radical nephroureterectomy with bladder cuff excision is the gold standard treatment for UTUC. Despite advances in diagnostic imaging and surgical techniques, the oncologic outcomes of five-year recurrence-free survival and cancer-specific survival vary greatly $[6,7]$. Some pathological variables including tumor stage, grade, lymph node metastasis status, lymphovascular invasion, tumor architecture, and multifocality are considered influential factors in determining survival [8]. However, these pathological characteristics still possess only a limited ability to predict outcomes. Moreover, the prognosis of patients with locally advanced or metastatic UTUC is poor, and chemotherapy lacks substantial benefits for high-risk cancer [9]. Therefore, specific biomarkers must be identified to predict outcomes and tailor personalized treatment and surveillance strategies.

$$
\text { MicroRNAs (miRNAs) are a family of short (22nt long, on average) small noncoding RNAs. }
$$
In UTUC, miRNAs that play a major role in tumor progression and cancer-specific survival were identified as regulators of gene expression, including oncogene and tumor suppressor genes. Signalling pathways modified by prognostic markers have been recognized as being involved in UTUC progression [10]. The regulatory roles of miRNAs in cell growth, apoptosis, autophagy, and aging have also been implicated [11].

Next-generation sequencing (NGS) is a useful tool for evaluating the genomic characteristics of various cancers, such as lung adenocarcinoma, renal cell carcinoma, colon cancer, and gastric 
90 cancer [12-14]. Through high-throughput sequencing, considerably high or low expression of 91 messenger RNAs and small RNAs can be identified. The purpose of this study was to discover 92 valuable biomarkers using a combination of bioinformatics tools including miRmap [15], Gene 93 Expression Omnibus (GEO), Oncomine [16], Ingenuity® Pathway Analysis (IPA) [17],

94 TargetScan, and miRDB [18] in our UTUC patient specimens focused on renal pelvis cancer.

95 Prior to the study findings were expected to reveal potential biomarkers and molecular 96 mechanisms to determine suitable diagnostic and therapeutic strategies.

97

98

Materials and Methods

100 Clinical UTUC specimen characteristics

We collected two pairs of tumor specimens and normal urothelial tissues from two UTUC patients in our hospital. These two patients had similar background characteristics, they were

103

104

105

106

107

108

109

110

111

112

113

114

115

116

117

118

119

120

121

122

123

124

125 both female with a diagnosis of high-grade renal pelvis UC. (Table 1). The Kaohsiung Medical University Hospital granted ethical approval to conduct the study within its facilities. This study was approved by our institutional review board (KMUHIRB-E(I)-20170018, KMUH107-7R56), and all patients provided written consent. To ensure the quality of the specimens, we collected them following strict procedures. After performing radical surgery, we collected specimens within $30 \mathrm{~min}$ then immediately preserved them in $\mathrm{a}-195.79^{\circ} \mathrm{C}$ liquid nitrogen container.

\section{Next-generation sequencing}

Using high-throughput sequencing and, NGS, we analyzed the whole genome, including mRNAs and microRNAs [19], and the sequencing depth is 30 million reads per sample. Two pairs of renal pelvis tumors and adjacent normal specimens were used in this study. Total RNA was extracted using TRIzol ${ }^{\circledR}$ Reagent (Invitrogen, Waltham, MA, USA) according to the manufacturer's instructions. Using an ND-1000 spectrophotometer (NanoDrop Technologies, Wilmington, DE, USA), the purified RNA was quantified at OD260nm and qualitatively analyzed using a Bioanalyzer 2100 (Agilent Technologies, Santa Clara, CA, USA) with an RNA 6000 LabChip kit (Agilent Technologies, Santa Clara, CA, USA). We cooperated with the Welgene Biotechnology Company (Welgene, Taipei, Taiwan) to assess RNA-seq and small RNA-seq using the official protocol of Illumina (San Diego, CA, USA). Raw sequences were obtained using the Illumina Pipeline software bcl2fastq v2.0.

After obtaining qualified reads, trimmomatics software was used to trim or remove the reads according to the quality score using TopHat/Cufflinks. A fold change $>2$ and fragments per kilobase million $($ FPKM) $>0.3$ were defined as significant expressions for mRNA analysis and a fold change $>2$ and reads per million (RPM) $>1$ were defined as significant expressions for miRNA analysis. The $p$-value was calculated by cuffdiff with non-grouped sample using "blind mode", in which all samples were treated as replicates of a single global "condition" and used to 
126 build one model for statistical testing. The Benjamini and Hochberg method was used to obtain $127 q$-values after adjusting for the false discovery rate. Therefore, we utilized these methods to 128 diminish the bias of workflow performance if the sample size was small as in previous research 129 [20]. We have deposited our data in the Gene Expression Omnibus (GEO) repository with the 130 accession number GSE 159824.

\section{1 miRmap database analysis for microRNA target predicting}

132 miRmap is a web application offering resource-predicting corresponding targets, including 133 miRNAs or genes. The miRmap library offers a complete range of features by combining thermodynamic, evolutionary, probabilistic, and sequence-based features to estimate potential candidate targets. By calculating miRNA-mRNA interaction prediction scores, and ranking them as miRmap scores, the most possible biomarker can be identified. In our study, we used final putative targets with miRmap scores $\geq 99.0$.

138

\section{Gene Expression Omnibus database and statistical analysis}

The GEO database is a web platform comprising microarrays, NGS, and other highthroughput functional genomics submitted by the research community. In the present study, we chose GSE19915 expression profiling using arrays, published in 2010. This database includes 144 bladder cancer samples and 12 normal samples analyzed using microarrays with the Strata gene Universal Human Reference RNA as the common reference sample. We then extracted raw data from GEO2R (https://www.ncbi.nlm.nih.gov/geo/geo2r/?acc=GSE19915) and calculated the expression differences using SPSS, version 19 (IBM Corp., Somers, NY, USA). The $p$ value was calculated using Student's t-test, and $p<0.05$ was considered statistically significant.

\section{Oncomine database analysis}

The Oncomine bioinformatics database collects over 18,000 cancer gene expression microarrays spanning various types of cancer including bladder cancer. Comparisons between clinical bladder cancer and normal specimens were performed using raw data on mRNA expression. A $p$ value $<1 \mathrm{E}-4$, fold change $>2$, and gene rank in the top $10 \%$ were defined as inclusion criteria. We then calculated the $p$ value using the Oncomine database with a two-sided Student's t-test.

\section{Ingenuity ${ }^{\circledR}$ Pathway Analysis}

$$
\text { IPA is a web-based software that analyzes omics data. An interaction network was presented }
$$
by integrating miRNAs and candidate genes. From the gene expression dataset, upstream factors and probable downstream effects were elucidated. TargetScan and miRDB analyses Using the online resources TargetScan and miRDB, we identified functional miRNAs from candidate gene targets. These computational tools can recognize the compensatory binding sites of matched targets from each miRNA.

\section{Western blot analysis}


162

163

164

165

166

167

168

169

170

171

172

173

174

175

176

177

178

179

180

181

182

183

184

185

186

187

188

189

190

191

192

193

194

195

196

197

The cell lysates $(30 \mu \mathrm{g})$ from urothelial cancer cell lines (RT4, BFTC905, J82, T24, UMUC3, BFTC 909 were obtained from Bioresource Collection and Research Center (BCRC), 5637 from ATCC, UMUC14 from European Collection of Authenticated Cell Cultures (ECACC)) were separated by $8 \%-12 \%$ SDS-PAGE gel and transferred onto a polyvinylidene difluoride membrane. Antibodies against human ZEB2 (1:5000 dilution, 14026-1-AP, Proteintech), CYBRD1 (1:5000 dilution, 26735-1-AP, Proteintech), PDE5A (1:5000 dilution, 22624-1-AP, Proteintech), and GAPDH (1:20000 dilution, 60004-1-Ig, Proteintech) were employed as primary antibodies. Rabbit or mouse IgG antibodies coupled with horseradish peroxidase were used as secondary antibodies. An enhanced chemiluminescence kit (Amersham) was used for protein detection (Amershan Biosciences, Piscataway, NJ, USA)

\section{Quantitative real time reverse transcriptase-PCR ( $q R T-P C R)$}

The mRNA expressions levels of PDE5A, CYBRD1, and ZEB2 were analyzed using qRTPCR. Total RNA was isolated from RT4, BFTC905, J82, T24, UMUC3, 5637, BFTC 909, UMUC14, and human tissues using an RNAzol kit (TEL-TEST Inc., Friendswoods, TX, USA). For reverse transcription, $5 \mu \mathrm{g}$ of total RNA was used with a Thermoscript reverse transcriptase (RT)-PCR system. Gene expression levels were amplified and detected using a Power SYBR ${ }^{\circledR}$ Green PCR Master Mix kit (Applied Biosystems; Life Technologies, Inc.) in a QuantStudio ${ }^{\mathrm{TM}} 3$ Real-Time PCR Detection System (Applied Biosystems; Life Technologies, Inc.).The PCR products were size-fractionated by electrophoresis in agarose gel, stained with TOOLS DNA View (TOOLS, Taipei, Taiwan) and photographed by ultraviolet light illumination.Primers used for target gene expression in the qRT-PCR are as follows: PDE5A (sense primer: 5'CGGCCCAAACCCTTAAAATT-3'; antisense primer: 5'-AGCGCTGTTTCCAGATCAGA-3'); CYBRD1(sense primer: 5'-CATGGTCACCGGCTTCGT-3'; antisense primer: 5'CAGGTCCACGGCAGTCTGTA-3'); ZEB2(sense primer: 5'-

ATATGGTGACACACAAGCCAGGGA-3'; antisense primer: 5'GTTTCTTGCAGTTTGGGCACTCGT-3')

\section{Immunohistochemistry}

Immunohistochemical staining of PDE5A, CYBRD1, and ZEB2 was performed on 4- $\mu$ m thick paraffin-embedded tissue sections. After deparaffinization, rehydration, antigen retrieval, and nonspecific binding blocking, tissue sections were incubated with an antibody against PDE5A (1:200 dilution, 22624-1-AP, Proteintech), CYBRD1 (1:200 dilution, 26735-1-AP, Proteintech), and ZEB2 (1:200 dilution, 14026-1-AP, Proteintech) at $37^{\circ} \mathrm{C}$ for $1 \mathrm{~h}$. The tissue sections were then rinsed twice with PBS for 2 min and then incubated with Mouse/Rabbit Probe HRP Labeling (BioTnA, TAHC03D) for $30 \mathrm{~min}$ at room temperature. They were then rinsed twice with PBS for 2 min and incubated in DAB peroxidase substrate solution (BioTnA) for 3 min, 
198

199

200

201

202

203

204

205

206

207

208

209

210

211

212

213

214

215

216

217

218

219

220

221

222

223

224

225

226

227

228

229

230

231

232

233

followed by a brief rinse with distilled water. The sections were counterstained with hematoxylin solution for $30 \mathrm{~s}$ (BioTnA, TA01NB). The results were recorded using a magnifier digital camera. Controls included omitting or preabsorbing the primary antibody and omitting secondary antibody.

\section{Results}

Identification of significant miRNA expression in renal pelvis cancer

To study potentially influential miRNA-mRNA interactions, miRNAs with a fold change $>2$ and FPKM $>0.3$ were selected. In total, 54 upregulated and 9 downregulated miRNAs were identified (Tables 2 and 3). The heatmap shows significant differentially expressed miRNAs with z-score $(\log 2)$ values between tumor and normal samples by using color clustering on the Morpheus web-tool. Blue represents downregulation and red represents upregulation (Figure 1).

\section{Identification of significant miRNA-mRNA interactions and genes}

To identify important miRNA-mRNA interactions and genes, we simultaneously analyzed our NGS results and miRmap putative genes. Using the NGS platform, 86 putative upregulated mRNAs and 225 putative downregulated mRNAs were identified. Using miRmap techniques to screen genes with miRmap scores $>99.0$, which indicated high predictive strength of repression, 115 putative upregulated target genes and 470 putative downregulated target genes were identified. The overlapping genes between miRNA putative targets and differentially expressed genes of our dataset were identified by Venn diagram analysis, and six potential genes, namely, PDE5A, RECK, ZEB2, NCALD, PLCXD3, and CYBRD1 had significant differences (Figure 2). To confirm whether the expression of these six identified genes was of clinical significance, we searched the GEO database for urothelial carcinoma or UTUC specimens. GSE19915: Subtype classification, grading, and outcome prediction of urothelial carcinomas using mRNA profiling combined with aCGHwere chosen for the array. This array compared urothelial carcinoma specimens and normal urothelial tissues [21]. When these six genes were searched for in this database, PDE5A, RECK, ZEB2, and CYBRD1 showed significant differences between tumor tissue and normal tissue (all $p<0.001$ ) (Figure 3). The results demonstrated low expression of PDE5A, RECK, ZEB2, and CYBRD1 in urothelial carcinoma tissue. The four downregulated genes with cooresponding miRNAs are listed in Table 4. We further investigated these genes using the Oncomine database, and the results also indicated lower expression of PDE5A, RECK, ZEB2, and CYBRD1 in bladder cancer tissue than in normal bladder mucosa (Figure 4).

\section{Identification of differentially expressed genes in UTUC and the potentially involved pathways}

To understand which pathways were potentially involved in the expression of the four 
234 identified genes, we used IPA software for analysis. The miRNA-gene network cell cycle

235 analysis revealed that all the aforementioned matched putative genes were associated with TP53.

236 Four networks including urothelial carcinoma, invasion, metastasis, and urogenital cancer, were

237 used to identify the affected genes. PDE5A, RECK, ZEB2, and CYBRD1 play essential roles by

238 interacting with other miRNAs and genes in urothelial carcinoma networks which are

239 represented by purple circles. RECK is an important factor in cancer invasion. RECK and ZEB2

240 are involved in cancer metastasis and are also associated with urogenital cancer networks (Figure

241 5A-D). Various miRNAs affect downstream mRNAs by interrupting cell cycle pathways and

242 promoting cancer progression. The network of PDE5A-related molecules obtained using the

243 Overlay tool in IPA included miR-19b-3p and WDR24.

244

245

\section{Identifying correlations among PDE5A, CYBRD1, and ZEB2 by an in vitro study}

246

We compared the expression of these genes in various UC cell lines (RT4, BFTC905, J82,

247 T24, UMUC3, 5637, BFTC 909, UMUC14) by western blot analysis and found decreased

248 expression of PDE5A in muscle-invasive UC cells compared with the RT4 cell line (Figure 6).

249 As shown in Figure 7, J82, T24, 5637, and BFTC 909 cells exhibited higher mRNA expression

250 of CYBRD1 and ZEB2 but lower expression of PDE5A than RT4 cells. Protein expression was

251

252

253

254

255

256

257

258

259

260

261

262

263

264

265

266

267

268

269 validated through histopathological analysis of our UTUC specimens, which revealed different levels and various localizations of PDE5A, CYBRD1, and ZEB2 present at different staining sites (Figure 8) in 200X magnification. To further clarify whether PDE5A is associated with UC, we evaluated PDE5A expression through real-time PCR from the paired UTUC tissues (normaltumor) of 20 patients, in total, 40 samples. The findings demonstrated lower expression of PDE5A in tumour specimens than in normal tissues. (Figure 9) The clinical characteristics of the 20 patients are shown in Table 5.

\section{Discussion}

UTUC originates from the urothelium and spans the whole urinary tract. Although bladder cancer is also derived from the urothelium, aggressive UTUC possesses many characteristics different from those of bladder UC. Overall, $60 \%$ of UTUCs present invasive patterns at diagnosis compared with $15 \%-25 \%$ of bladder tumors [22]. However, the factors that contribute to the differences in UTUC rates between Western regions and Taiwan remain unclear. It is crucial to identify novel biomarkers that can compensate for or replace current surveillance techniques. We initially combined high-throughput NGS analysis with bioinformatics tools to select critical miRNAs or genes including PDE5A, RECK, CYBRD1, and ZEB2 for further comprehensive research.

Phosphodiesterase 5A (PDE5A), a gene responsible for coding cGMP-binding and cGMP- 
270 specific phosphodiesterase, is a member of the cyclic nucleotide phosphodiesterase family. The

271 function of PDE5A is to degrade cGMP to 5'-GMP. Some studies have demonstrated that the

272 PDE5 inhibitor sildenafil which is clinically used for treating erectile dysfunction diseases,

273 promotes melanoma cell invasion and growth and increases melanoma risk [23-24]. Another

274 study revealed that the cGMP pathway may have crosstalk with the MAPK pathway to affect the

275 pathophysiology and therapy of melanoma. PDE5 inhibitors enhance melanoma cell growth by

276 activating cGMP pathways both in vitro and in vivo, thus indicating a link between PDE5 and

277 cGMP pathways in melanoma cells [25]. The cGMP pathway also affects various reactions in

278 tumor microenvironments, such as blood supply, angiogenesis, inflammation, and immune

279 reactions [26]. Another theory proposed that PDE5A is related to hypoxia in tumor

280 microenvironments. When tumor cells are under hypoxic conditions, microphthalmia-associated

281 transcription factors are inhibited, reducing the expression of PDE5A, and promoting the

282 proliferation and metastatic phenotype of melanoma [27]. Based on previous research and

283

284

285

286

287

288

289

290

291

292

293

294

295

296

297

298

299

300

301

302

303

304

305 assumptions about correlations between PDE5A and melanoma, PDE5A — selected from our NGS and bioinformatics analyses - may be considered a crucial factor influencing UTUC tumor cell progression. In our in vitro study, PDE5A demonstrated lower mRNA and protein expression in advanced UC cell lines. Results from the comparison of normal tissue and tumor specimens from 20 patients also revealed lower expression in tumor specimens. Therefore, we hypothesized that PDE5A plays the role of a tumor suppressor in UC.

Reversion-inducing cysteine-rich protein with kazal motifs (RECK), which is a tumor suppressor, regulates matrix metalloproteinases (MMPs) by breaking down the extracellular matrix (ECM). The integrity of the ECM, which is maintained by RECK, is associated with tumor invasion and metastasis. When RECK inhibits MMPs, the basement membrane remains intact and inhibits tumor angiogenesis to prevent blood vessels from supplying to tumor cells. Studies have mentioned the role of RECK in tumors including lung, breast, prostate, oral, digestive tract, liver, and pancreatic cancers [28]. Because evidence suggests that the RECK gene is related to oncogenesis, it may be a therapeutic target. If a therapeutic means of enhanceing RECK expression is developed, it may be valuable in improving prognosis and impeding tumor progression. Another theory regarding RECK relates to hypoxic microenvironments, which interact with hypoxia-inducible factor (HIF)-1 $\alpha$ prompting RECK downregulation with histone deacetylase (HDAC)1 to silence the RECK gene [29].

In addition, RECK promotes angiogenesis through the mediation of the vascular endothelial growth factor, which stimulates angiogenesis and is essential for development and differentiation [30]. RECK affects not only tumor progression but also tissue architecture remodelling in embryonic development. Although the influence of RECK has been studied in various cancers, its role in UTUC remains unclear. Further investigation is required to identify potential 
306

307

308

309

310

311

312

313

314

315

316

317

318

319

320

321

322

323

324

325

326

327

328

329

330

331

332

333

334

335

336

337

338

339

340

341

prognostic or therapeutic targets of RECK in UTUC.

Cytochrome $b$ reductase 1 (CYBRD1) is a member of the cytochrome $b(561)$ family and is distributed mainly within duodenum cells. The function of CYBRD is the reduction of $\mathrm{Fe}^{3+}$ to $\mathrm{Fe}^{2+}$, regulation of ferrous metabolism, absorption, and transportation. A study on breast cancer detected duodenal cytochrome $b$ (DCYTB) on the surface of breast cancer epithelial cells. The expression of DCYTB is associated with breast cancer survival, high-grade tumors demonstrated significantly lower expression of DCYTB, and at high expression levels of DCYTB, patients had better prognoses. The expression of this gene also reduces tumor cell adhesion and metastasis by diminishing the activation of focal adhesion kinase [31]. Western blotting and qPCR analysis of cultured cells revealed that activation of the DCYTB gene is also regulated by Hif-2 $\alpha$ [32]. Further research is necessary to clarify whether the gene is a novel biomarker for UTUC.

Further searching for predictive upstream regulatory miRNAs from miRmap, TargetScan, target sites of miR-181c-5p on PDE5A were positioned 3767-3773 and 3986-3992 of PDE5A 3' UTR. The role of miR-181c in cancer has also been validated in pancreatic cancer. Chen et al. demonstrated that high expression of miR-181c was associated with poor prognosis and survival. Furthermore, it induced cancer cell chemoresistance by inactivating the Hippo signaling pathway [33]. A recent pancreatic cancer-bearing mouse model confirmed the hypothesis that it reverses the effect of multidrug resistance by downregulating miR-181c-5p [34]. Another candidate miRNA, miR-200c-3p which is at positions 126-132 and 1207-1214 of RECK 3' UTR, has been investigated in several cancer types and is associated with cancer progression and metastasis by regulating epithelial-mesenchymal transition. However, whether the effect of regulatory mechanisms can translate to clinical survival in patients with UTUC still requires elucidation [35].

Due to the advent of NGS technologies over the past few years, identification of specific target genes has become a feasible strategy for stratifying the risk of disease and offering a targeted oncological treatment. A recent review article discussed the molecular characterization of UTUC using NGS analysis and demonstrated that different gene expression levels or mutational profiles present different risks of cancer, which can be target for prognostic or therapeutic strategies. If gene expression is the next era of precision therapy, combining expression profiling with potential candidate genes, including our NGS dataset, may increase the accuracy to predict cancer survival or progression. Furthermore, it will also be interesting to conduct further research on different patient characteristics between countries and ethnicities [36].

In conclusion, our study revealed that PDE5A, RECK, ZEB2, and CYBRD1 are predictive candidate genes in UTUC through NGS and bioinformatics analyses. The expression of these genes was lower in cancer specimens than in normal tissues. Research indicates that PDE5A, RECK, and CYBRD1are tumor suppressor genes involved in angiogenesis, hypoxia 
342 microenvironment, and metabolism. These candidate genes are associated with cancer

343 progression and survival. In vitro studies further clarified the expression of PDE5A, CYBRD1, 344 and ZEB2. PDE5A is also a potential tumor suppressor gene, as identified by comparing the 345 expression levels between normal and tumor specimens from 20 patients. Future studies with 346 large sample size and combined candidate gene identification will be crucial to translate these 347 findings to precision medicine strategies.

348

349 Compliance with Ethical Standards:

350 Funding: This study was not funded.

351 Conflicts of interests: The authors declare no competing financial interests.

352 Ethical approval: This study was approved by our institutional review board (KMUHIRB-E(I)$35320170018)$

354 Informed consent: All included patients provided written informed consent.

\section{Acknowledgements}

356 This study was supported by grants from the Ministry of Science and Technology (MOST 106357 2314-B-037-029), Kaohsiung Medical University Hospital (KMUH 106-6R53), Kaohsiung 358 Municipal Ta-Tung Hospital (kmtth-108-R003) and partially by Kaohsiung Medical University 359 Grant (KMU-KI109002), Regenerative Medicine and Cell Therapy Research Center Grant 360 (KMU-TC108A02), Cohort Research Center (KMU-TC108B07).

\section{Author's Contribution}

362 HY Lee: Protocol/project development, Data management, Manuscript writing

363 CC Li: Protocol/project development, Data collection

364 WM Li: Protocol/project development, Data collection

365 YL Hsu: Protocol/project development, Data analysis

366 HC Yeh: Protocol/project development, Data analysis 
367 HL Ke: Protocol/project development, Data collection

368 BW Yeh: Protocol/project development

369 CN Huang: Protocol/project development, Data collection

370 CF Li: Protocol/project development

371 WJ Wu: Protocol/project development, Data collection

372 PL Kuo: Protocol/project development, Data analysis

373

374 Reference:

375 1. Li WM, Huang CN, Ke HL, Li CC,Wei YC, Yeh HC, Chang LL, Huang CH, Liang PI, Yeh

376 BW, Chan TC, Li CF, Wu WJ. MCM10 overexpression implicates adverse prognosis in

377 urothelial carcinoma. Oncotarget. 2016;7:77777-77792.

378 2. Shen CH, Chiou HY, Tung MC, Wu CC, Kao WT, Wang YH, Juang GD. Clinical and

379 demographic characteristics among patients with urothelial carcinomas of the upper urinary

380 tract and bladder in Taiwan. J Chin Med Assoc. 2017;80:563-568

381 3. Shahrokh F Shariat, Ricardo L Favaretto, Amit Gupta, Hans-Martin Fritsche, Kazumasa

382

383

384

385

386

387

388

389

390

391

392

393

394

395

396

397

398

399

Matsumoto, Wassim Kassouf, Thomas J Walton, Stefan Tritschler, Shiro Baba, Kazuhito

Matsushita, Patrick J Bastian, Juan I Martínez-Salamanca, Christian Seitz, Armin

Pycha, Wolfgang Otto, Pierre I Karakiewicz, Vincenzo Ficarra, Giacomo Novara. Gender differences in radical nephroureterectomy for upper tract urothelial carcinoma. World $\mathrm{J}$ Urol. 2011;29:481-6.

4. Pierre Colin, Philippe Koenig, Adil Ouzzane, Nicolas Berthon, Arnauld Villers, Jacques Biserte, Morgan Rouprêt. Environmental factors involved in carcinogenesis of urothelial cell carcinomas of the upper urinary tract. BJU Int. 2009;104:1436-40.

5. Miyazaki J, Nishiyama H. Epidemiology of urothelial carcinoma. Int J Urol. 2017;24:730734.

6. Vitaly Margulis, Shahrokh F Shariat, Surena F Matin, Ashish M Kamat, Richard Zigeuner, Eiji Kikuchi, Yair Lotan, Alon Weizer, Jay D Raman, Christopher G Wood Outcomes of radical nephroureterectomy: a series from the Upper Tract Urothelial Carcinoma Collaboration. Cancer 2009;115:1224-33.

7. Benoit Peyronnet, Thomas Seisen, Jose-Luis Dominguez-Escrig, Harman Max Bruins, Cathy Yuhong Yuan, Thomas Lam, Steven Maclennan, James N'dow, Marko Babjuk, Eva Comperat, Richard Zigeuner, Richard J. Sylvester, Maximilian Burger, Hugh Mostafid, Bas W.G. van Rhijn, Paolo Gontero, Joan Palou, Sharokh F. Shariat, Morgan Roupret.

PeerJ reviewing PDF | (2020:10:54841:2:0:NEW 27 Mar 2021) 
400

401

402

403

404

405

406

407

408

409

410

411

412

413

414

415

416

417

418

419

420

421

422

423

424

425

426

427

428

429

430

431

432

433

434

435

Oncological Outcomes of Laparoscopic Nephroureterectomy Versus Open Radical Nephroureterectomy for Upper Tract Urothelial Carcinoma: An European Association of Urology Guidelines Systematic Review. Eur Urol Focus. 2017 Nov 15. pii: S24054569(17)30240-7

8. Hsiang-Ying Lee, Ching-Chia Li, Chun-Nung Huang, Hung-Lung Ke, Wei-Ming Li, Peir-In Liang, Sheau-Fang Yang, Hung-Pin Tu, Wen-Jeng Wu, Hsin-Chih Yeh. Prognostic significance of lymphovascular invasion in upper urinary tract urothelial carcinoma is influenced by tumour location. Ann Surg Oncol. 2015;22:1392-400.

9. Nilay Patel, Manit Arya, Asif Muneer, Tom Powles, Mark Sullivan, John Hines, John Kelly. Molecular aspects of upper tract urothelial carcinoma. Urol Oncol. 2014;32:28.e11-20.

10. Laura Izquierdo, Ruth Montalbo, Mercedes Ingelmo-Torres, Carme Mallofré, Miguel Ramírez-Backhaus, Jose Rubio, Antoine G. Van der Heijden, Ewout Schaafsma, Antonio Lopez-Beltran, Ana Blanca, Nathan Lawrentschuk, Antonio Alcaraz and Lourdes Mengual. Prognostic microRNAs in upper tract urothelial carcinoma: multicenter and international validation study. Oncotarget. 2017; 8: 51522-51529.

11. Eun Jung Sohn. MicroRNA 200c-3p regulates autophagy via upregulation of endoplasmic reticulum stress in PC-3 cells. Cancer Cell Int. 2018; 18: 2.

12. Ya-Ling Hsu, Jen-Yu Hung, Yen-Lung Lee, Feng-Wei Chen, Kuo-Feng Chang, Wei-An Chang, Ying-Ming Tsai, Inn-Wen Chong and Po-Lin Kuo. Identification of novel gene expression signature in lung adenocarcinoma by using next-generation sequencing data and bioinformatics analysis. Oncotarget. 2017;8:104831-104854.

13. Omar Youssef, Virinder Sarhadi, Homa Ehsan, Tom Böhling, Monika CarpelanHolmström, Selja Koskensalo, Pauli Puolakkainen, Arto Kokkola, Sakari Knuutila. Gene mutations in stool from gastric and colorectal neoplasia patients by next-generation sequencing. World J Gastroenterol. 2017;23:8291-8299.

14. Chen SC, Chen FW, Hsu YL, Kuo PL. Systematic Analysis of Transcriptomic Profile of Renal Cell Carcinoma under Long-Term Hypoxia Using Next-Generation Sequencing and Bioinformatics. Int J Mol Sci. 2017;18. pii: E2657.

15. Vejnar CE, Blum M, Zdobnov EM. miRmap web: Comprehensive microRNA target prediction online. Nucleic Acids Res. 2013;41(Web Server issue):W165-8.

16. Daniel R Rhodes, Shanker Kalyana-Sundaram, Vasudeva Mahavisno, Radhika Varambally, Jianjun Yu, Benjamin B Briggs, Terrence R Barrette, Matthew J Anstet, Colleen KinceadBeal, Prakash Kulkarni, Sooryanaryana Varambally, Debashis Ghosh, and Arul M Chinnaiyan. Oncomine 3.0: Genes, Pathways, and Networks in a Collection of 18,000 Cancer Gene Expression Profiles. Neoplasia. 2007; 9: 166-180.

17. Zhao M, Liu D, Qu H. Systematic review of next-generation sequencing simulators:

Peer) reviewing PDF | (2020:10:54841:2:0:NEW 27 Mar 2021) 
436

437

438

439

440

441

442

443

444

445

446

447

448

449

450

451

452

453

454

455

456

457

458

459

460

461

462

463

464

465

466

467

468

469

470

471

computational tools, features and perspectives. Brief Funct Genomics. 2017; 16:121-128.

18. Nathan Wong and Xiaowei Wang. miRDB: an online resource for microRNA target prediction and functional annotations. Nucleic Acids Res. 2015; 43(Database issue): D146D152.

19. Andreas Krämer, Jeff Green, Jack Pollard, Jr, and Stuart Tugendreich.Causal analysis approaches in Ingenuity Pathway Analysis. Bioinformatics. 2014; 30: 523-530.

20. Alyssa Baccarella, Claire R. Williams, Jay Z. Parrish \& Charles C. Kim. BMC Bioinformatics. 2018;19:423

21. David Lindgren, Attila Frigyesi, Sigurdur Gudjonsson, Gottfrid Sjödahl, Christer Hallden, Gunilla Chebil, Srinivas Veerla, Tobias Ryden, Wiking Månsson, Fredrik Liedberg, Mattias Höglund. Combined gene expression and genomic profiling define two intrinsic molecular subtypes of urothelial carcinoma and gene signatures for molecular grading and outcome. Cancer Res 2010;70:3463-72.

22. Morgan Rouprêt, Marko Babjuk, Eva Compérat, Richard Zigeuner, Richard J Sylvester, Maximilian Burger, Nigel C Cowan, Paolo Gontero, Bas W G Van Rhijn, A Hugh Mostafid, Joan Palou, Shahrokh F Shariat. European Association of Urology Guidelines on Upper Urinary Tract Urothelial Carcinoma: 2017 Update. Eur Urol. 2018;73:111-122.

23. Imanol Arozarena, Berta Sanchez-Laorden, Leisl Packer, Cristina Hidalgo-Carcedo, Robert Hayward, Amaya Viros, Erik Sahai, Richard Marais. Oncogenic BRAF induces melanoma cell invasion by downregulating the cGMP-specific phosphodiesterase PDE5A. Cancer Cell 2011;19: 45-57.

24. Li, W.Q., Qureshi, A.A., Robinson, K.C., and Han, J. Sildenafil use and increased risk of incident melanoma in US men: a prospective cohort study. JAMA Intern. Med. 2014;174, 964-970.

25. Sandeep Dhayade, Susanne Kaesler, Tobias Sinnberg, Hyazinth Dobrowinski, Stefanie Peters, Ulrike Naumann, He Liu, Robert E Hunger, Martin Thunemann, Tilo Biedermann, Birgit Schittek, Hans-Uwe Simon, Susanne Feil, Robert Feil. Sildenafil Potentiates a cGMP-Dependent

Pathway to Promote Melanoma Growth. Cell Rep. 2016;14:2599-610.

26. Fajardo, A.M., Piazza, G.A., and Tinsley, H.N. The role of cyclic nucleotide signaling pathways in cancer: targets for prevention and treatment. Cancers(Basel). 2014;6:436-458

27. Houslay MD. Melanoma, Viagra, and PDE5 Inhibitors: Proliferation and metastasi Metastasis.Trends Cancer. 2016;2:163-165.

28. Nagini S. RECKing MMP: Relevance of Reversion-inducing Cysteine-rich Protein with Kazal Motifs as a Prognostic Marker and Therapeutic Target for Cancer (A Review). 
472

473

474

475

476

477

478

479

480

481

482

483

484

485

486

487

488

489

490

491

492

493

494

495

496

497

498

499

500

501

502

503

504

505

506

507

Anticancer Agents Med Chem. 2012;12:718-25.

29. Zhu J, Ling Y, Xu Y, Lu M, Liu Y, Zhang C.Promoter hypermethylation of the RECK gene is associated with its low expression and poor survival of esophageal squamous cell carcinoma. Oncol Lett. 2017;13:1911-1918.

30. Alexius-Lindgren M, Andersson E, Lindstedt I, Engström W. The RECK gene and biological malignancy--its significance in angiogenesis and inhibition of matrix metalloproteinases. Anticancer Res. 2014;34:3867-73.

31. David J. Lemler, Miranda L. Lynch, Lia Tesfay, Zhiyong Deng, Bibbin T. Paul, Xiaohong Wang, Poornima Hegde, David H. Manz, Suzy V. Torti and Frank M. Torti. DCYTB is a predictor of outcome in breast cancer that functions via iron-independent mechanisms. Breast Cancer Res. 2017 Mar 7;19:25.

32. Luo X, Hill M, Johnson A, Latunde-Dada GO. Modulation of Dcytb (Cybrd 1) expression and function by iron, dehydroascorbate and Hif- $2 \alpha$ in cultured cells.Biochim Biophys Acta. 2014;1840:106-12.

33. Chen M., Wang M., Xu S., Guo X., and Jiang J.: Upregulation of miR-181c contributes to chemoresistance in pancreatic cancer by inactivating the Hippo signaling pathway. Oncotarget 2015; 6: pp. 44466-44479

34. Zhi-Qiang Gao, Jun-Feng Wang, De-Hua Chen, Xue-Song Ma, Wu Yang, Tang Zhe, XiaoWei Dang. Long non-coding RNA GAS5 antagonizes the chemoresistance of pancreatic cancer cells through down-regulation of miR-181c-5p. Biomed Pharmacother. 2018;97:809817.

35. Xuegang Wang, Xuanyu Chen, Rong Wang, Pei Xiao, Zhenghong Xu, Li Chen, Weiwei Hang, Anming Ruan, Hongmei Yang, Xiaoping Zhang. microRNA-200c modulates the epithelial-to-mesenchymal transition in human renal cell carcinoma metastasis. Oncol Rep. 2013;30:643-650.

36. Melanie R Hassler, Freddie Bray, James W F Catto, Arthur P Grollman, Arndt Hartmann, Vitaly Margulis, Surena F Matin, Morgan Roupret, John P Sfakianos, Shahrokh F Shariat, Bishoy M Faltas. Molecular Characterization of Upper Tract Urothelial Carcinoma in the Era of Next-generation Sequencing: A Systematic Review of the Current Literature. Eur Urol. 2020;78:209-220

Figure Legends

Figure 1. Heatmap exhibiting significant difference expression of miRNAs with z-score $(\log 2)$ values between tumor and normal samples. (A) miRNA up expression (B) miRNA down expression 
508

509 Figure 2. Identification of potential candidate genes through a combination of next-generation 510 sequencing (NGS) and miRmap databases. (A) NGS analysis identified 86 upregulated genes 511 and 115 putative genes using miRmap analysis. No potential candidates for upregulated genes

512 were identified after interaction. (B) NGS analysis identified 225 downregulated genes and 470

513 putative genes through miRmap analysis. Six significant differentially downregulated candidate 514 genes were recognized.

515

516 Figure 3.A related array (Gene Expression Atlas accession: GSE19915) was used to analyze 517 significant expression of A: PDE5A, B: RECK, C: ZEB2, and D: CYBRD1 between urothelial 518 carcinoma specimens and urothelial tissues (all $p<0.001)$.

519

520

521

522

523

524

525

526

527

528

529

530

531

532

533

534

535

536

537

538

539

540

541

542

543

Figure 4. Analysis of (A) PDE5A, (B) RECK, (C) ZEB2, and (D) CYBRD1 expression in bladder cancer compared with normal tissue using the Oncomine database.

Figure 5. Network prediction for PDE5A, RECK, ZEB2, and CYBRD1 involved in urothelial carcinoma analyzed using Ingenuity Pathway Analysis database. (A) The four candidate genes were associated with TP53. (B) From the cancer invasion network, RECK-indicated by a purple circle - is a potential gene associated with cancer invasion. (C) From the cancer metastasis network, RECK and ZEB2 — indicated by purple circles_play critical roles in cancer metastasis. (D) From the urogenital cancer network, RECK and ZEB2 - indicated by purple circles-were demonstrated to be involved in urogenital cancer pathways.

Figure 6. Protein expression analyzed using western blot analysis. Antibodies against human ZEB2, CYBRD1, PDE5A, and GAPDH were employed as primary antibodies.

Figure 7. mRNA expression of (A) PDE5A, (B) CYBRD1, and (C) ZEB2 in UC and UTUC cells. J82, T24, 5637, and BFTC 909 cells exhibited higher expression of CYBRD1 and ZEB2 but lower expression of PDE5A than RT4 cells. (D) Expression of fold of control levels.

Figure 8. Illustration of histopathological analysis of sections from surgical resected human UTUC tissue (A). It shows different levels of localization for PDE5A, CYBRD1, and ZEB2 staining in 200X magnification. Scale bar, $100 \mu \mathrm{m}$. (B) Positive control= breast tumor tissue with higher PDE5A expression. (C) Reagent control=without primary antibody.

Figure 9. Paired UTUC tissues (normal and tumor) from 20 patients were evaluated for PDE 5A

Peer] reviewing PDF | (2020:10:54841:2:0:NEW 27 Mar 2021) 
544 expression through real-time PCR. GAPDH was used as an internal control. The relative 545 expression of PDE5 in tumors was significantly lower than that in normal UTUC tissues (2.55546 fold, $p<0.05)$.

547 
Figure 1

Heatmap exhibiting significant difference expression of miRNAs

(A) miRNA up expression (B) miRNA down expression 
A

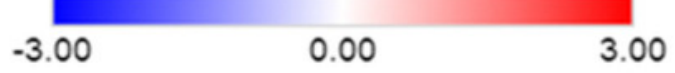

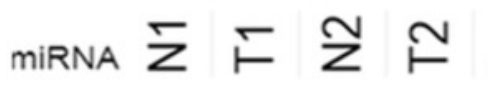

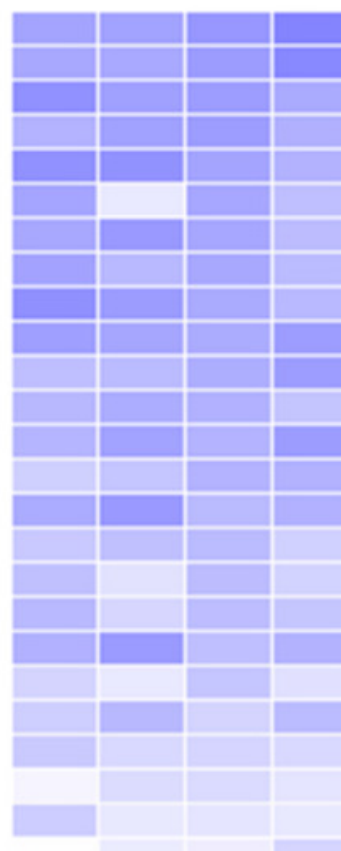

hsa-miR-128-3p

hsa-miR-128-3p

hsa-miR-200a-5

hsa-miR-181c-3

hsa-miR-452-5p

hsa-miR-210-3p

hsa-miR-1307-3

hsa-miR-130b-3

hsa-miR-149-5p

hsa-miR-106b-5

hsa-miR-421

hsa-miR-345-5p

hsa-miR-454-3p

hsa-miR-1307-5

hsa-miR-1260b

hsa-miR-181d-5

hsa-miR-20a-5p

hsa-miR-17-5p

hsa-miR-1260a

hsa-miR-425-5p

hsa-miR-34a-5p

hsa-miR-429

hsa-miR-301a-3

hsa-miR-200a-3

hsa-miR-941

hsa-miR-941

hsa-miR-941

hsa-miR-941

hsa-miR-941

hsa-miR-769-5p

hsa-miR-335-3p

hsa-miR-222-3p

hsa-miR-203a-3

hsa-let-7d-5p

hsa-miR-181c-5

hsa-miR-200b-3

hsa-miR-93-5p

hsa-miR-200c-3

hsa-miR-221-3p

hsa-miR-151a-5

hsa-miR-183-5p

hsa-miR-205-5p

hsa-miR-21-3p

hsa-miR-98-5p

hsa-let-7b-5p

hsa-miR-151a-3

hsa-miR-141-3p

hsa-miR-182-5p

hsa-miR-92a-3p

hsa-miR-92a-3p

hsa-miR-191-5p

hsa-let-7a-5p

hsa-let-7a-5p

hsa-let-7a-5p
B $-3.00$

0.00

3.00

miR

miR

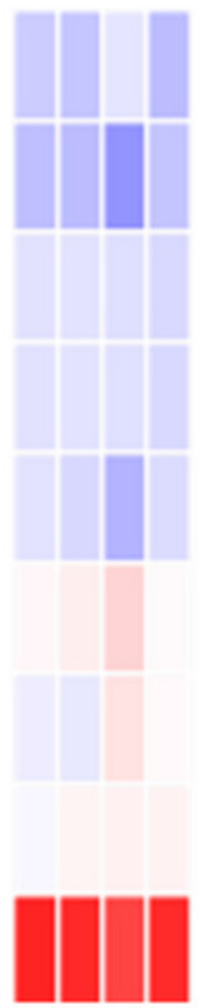

hsa-miR-99a-5p

hsa-miR-145-3p

hsa-miR-125b-5p

hsa-miR-125b-5p

hsa-miR-145-5p

hsa-miR-100-5p

hsa-miR-451a

hsa-let-7c-5p

hsa-miR-143-3p

miRNA down expression

\section{miRNA up expression}




\section{Figure 2}

Identification of potential candidate genes through a combination of next-generation sequencing (NGS) and miRmap databases

(A) NGS analysis identified 86 upregulated genes and 115 putative genes using miRmap analysis. No potential candidates for upregulated genes were identified after interaction. (B) NGS analysis identified 225 downregulated genes and 470 putative genes through miRmap analysis. Six significant differentially downregulated candidate genes were recognized.

A

NGS: up-regulated genes

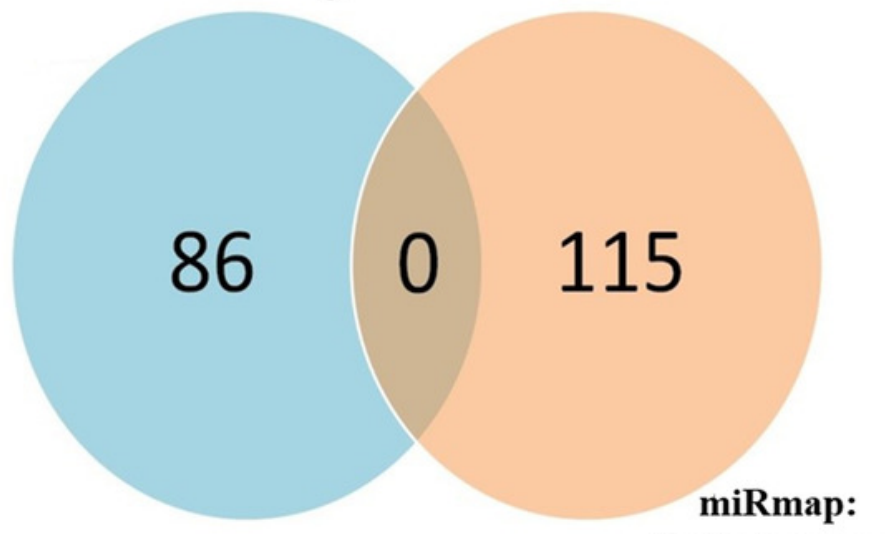

Putative genes
B

NGS: down-regulated genes

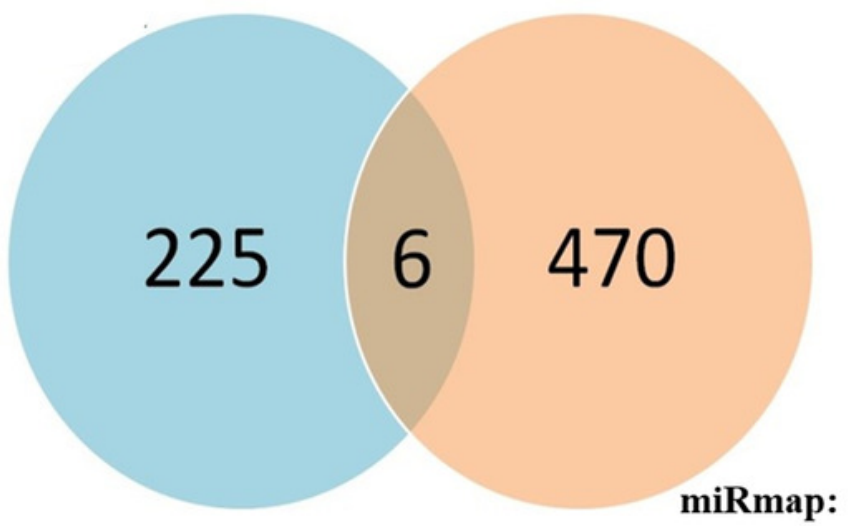

Putative genes 
Figure 3

A related array (Gene Expression Atlas accession: GSE19915) was used to analyze significant expression

Expression of A: PDE5A, B: RECK, C: ZEB2, and D: CYBRD1 between urothelial carcinoma specimens and urothelial tissues

A PDE5A

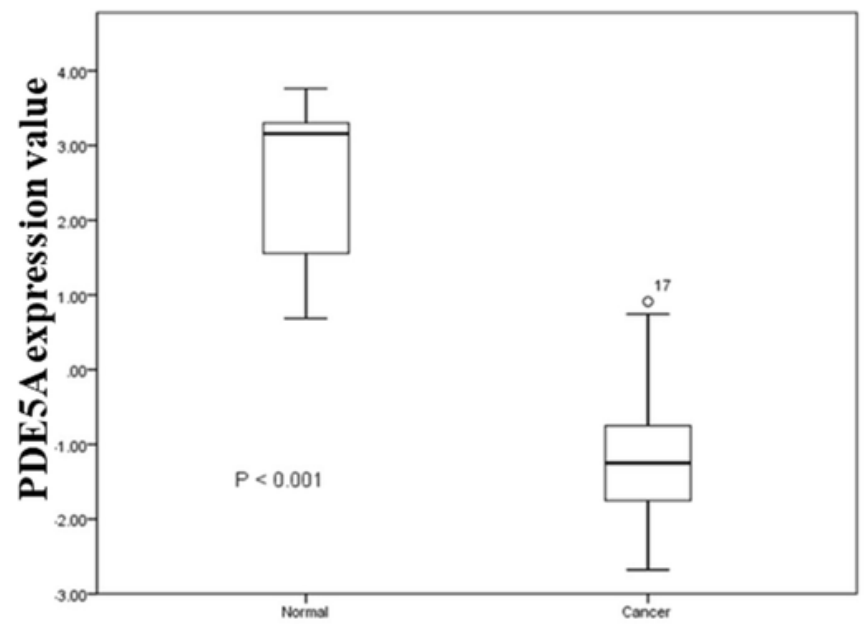

C ZEB2

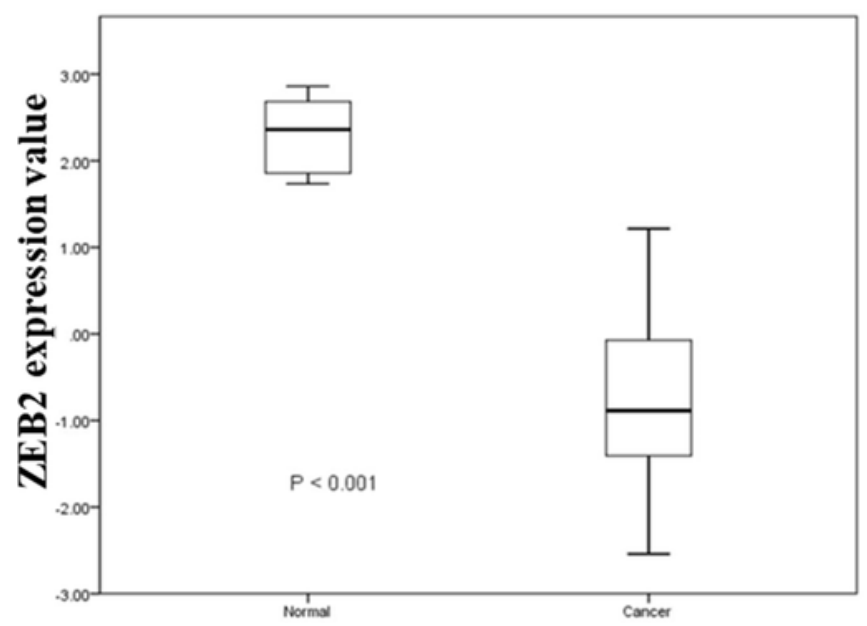

B RECK

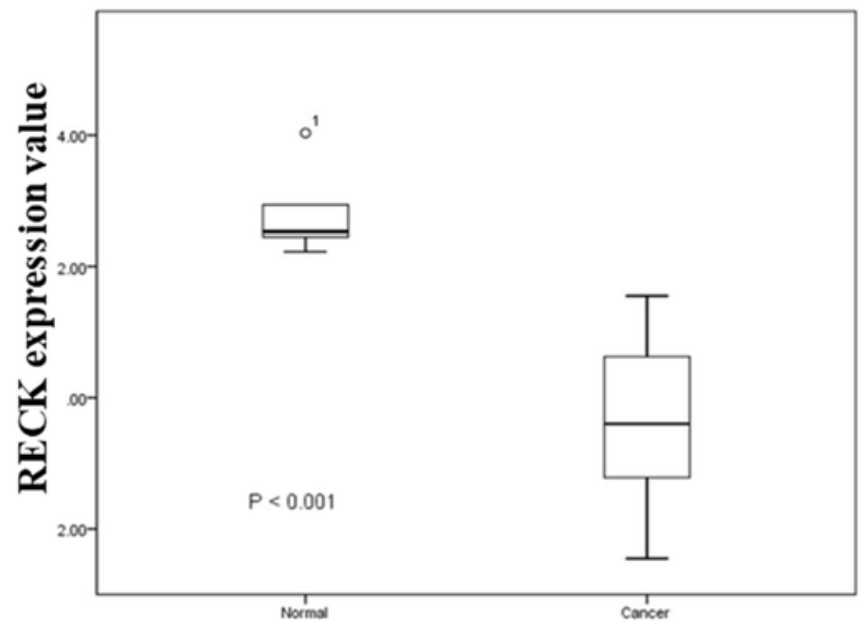

D CYBRD1

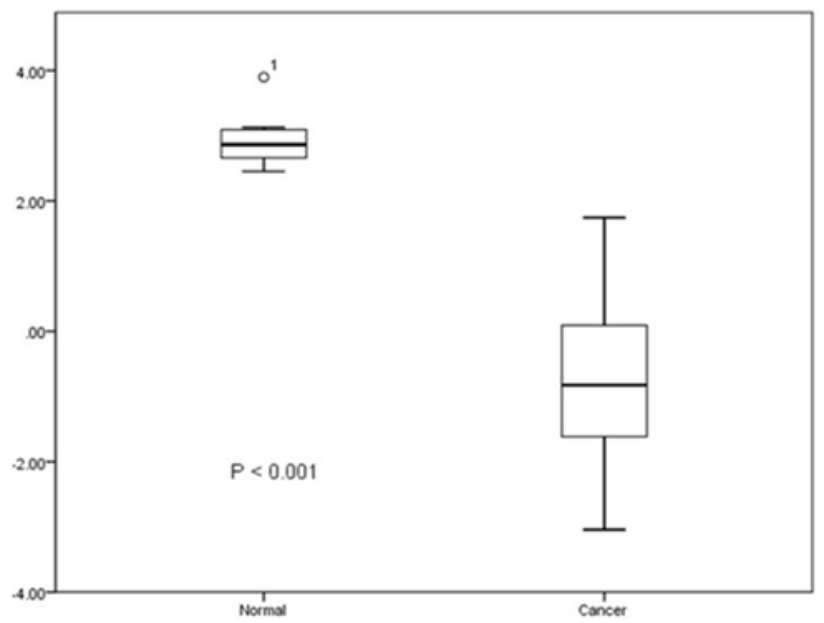


Figure 4

Expression in bladder cancer compared with normal tissue using the Oncomine database.

Analysis of (A) PDE5A, (B) RECK, (C) ZEB2, and (D) CYBRD1 expression
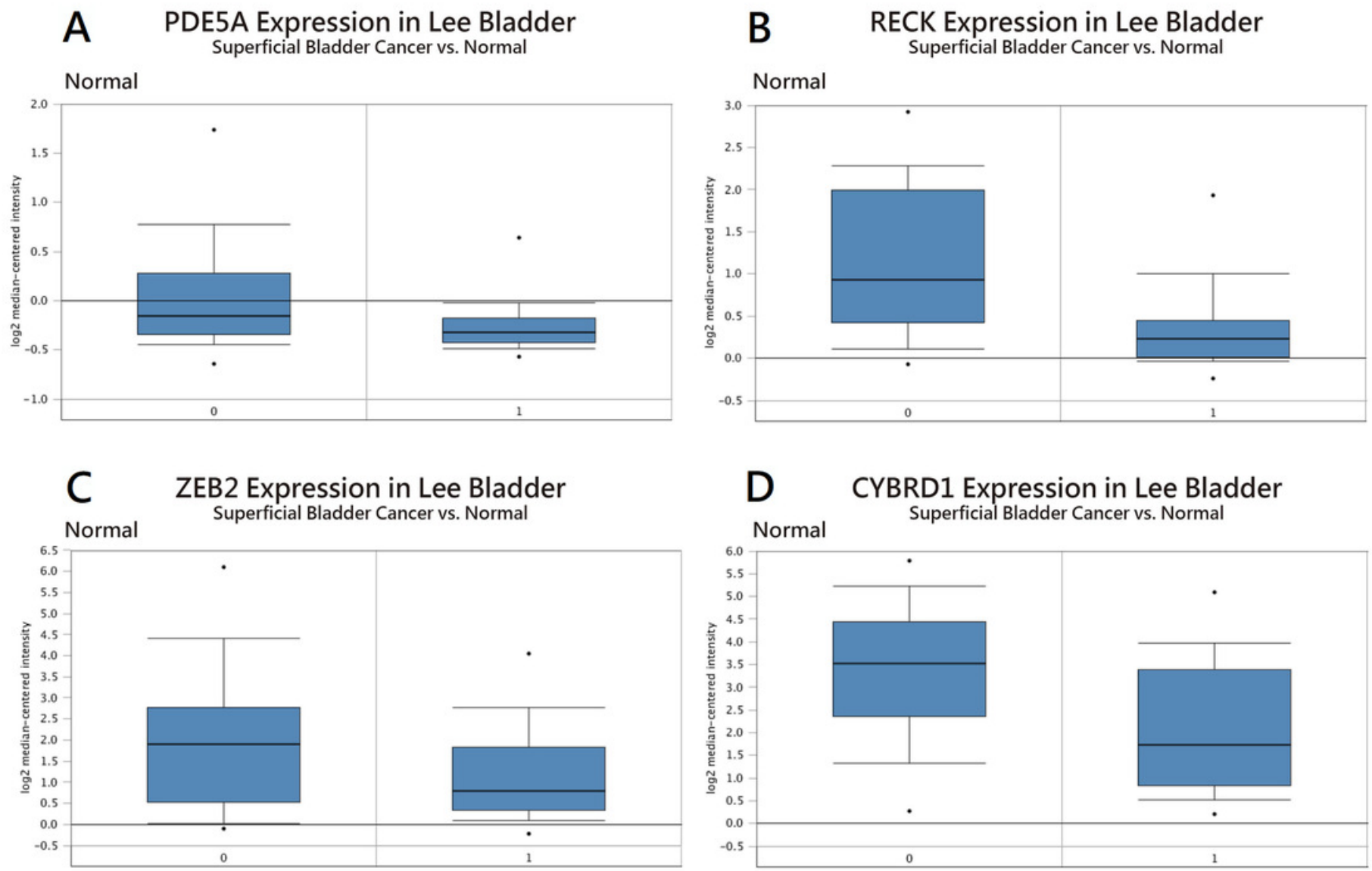


\section{Figure 5}

Network prediction for PDE5A, RECK, ZEB2, and CYBRD1 involved in urothelial carcinoma analyzed using Ingenuity Pathway Analysis database.

Ingenuity Pathway Analysis database. (It is a public database) (A) The four candidate genes were associated with TP53. (B) From the cancer invasion network, RECK-indicated by a purple circle-is a potential gene associated with cancer invasion. (C) From the cancer metastasis network, RECK and ZEB2-indicated by purple circles-play critical roles in cancer metastasis. (D) From the urogenital cancer network, RECK and ZEB2-indicated by purple circles-were demonstrated to be involved in urogenital cancer pathways. 


\section{Network 1: Observation 1: UTUC mRNA down: Observation 1}

A

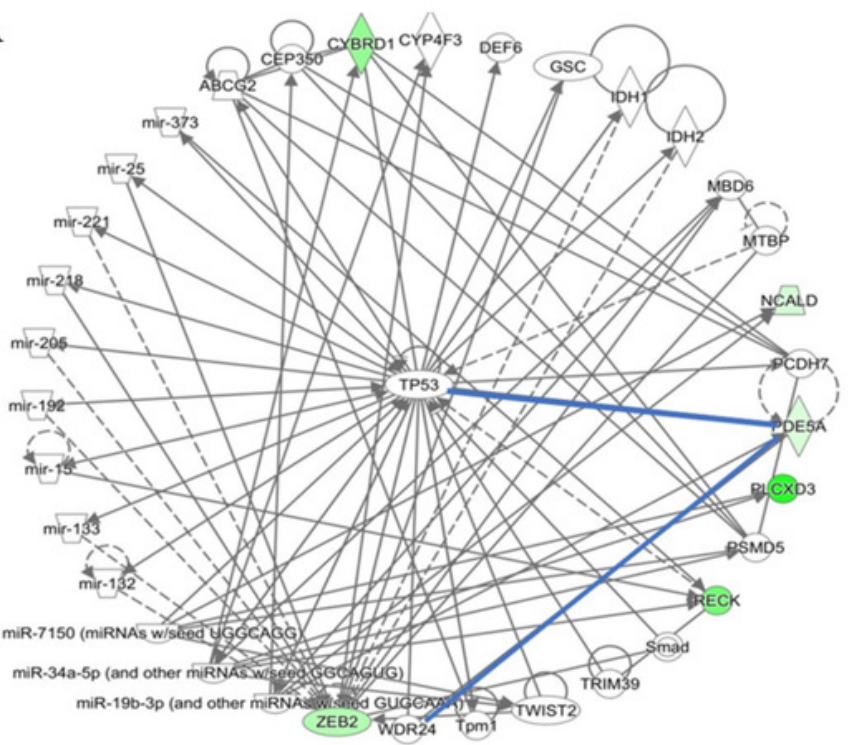

C

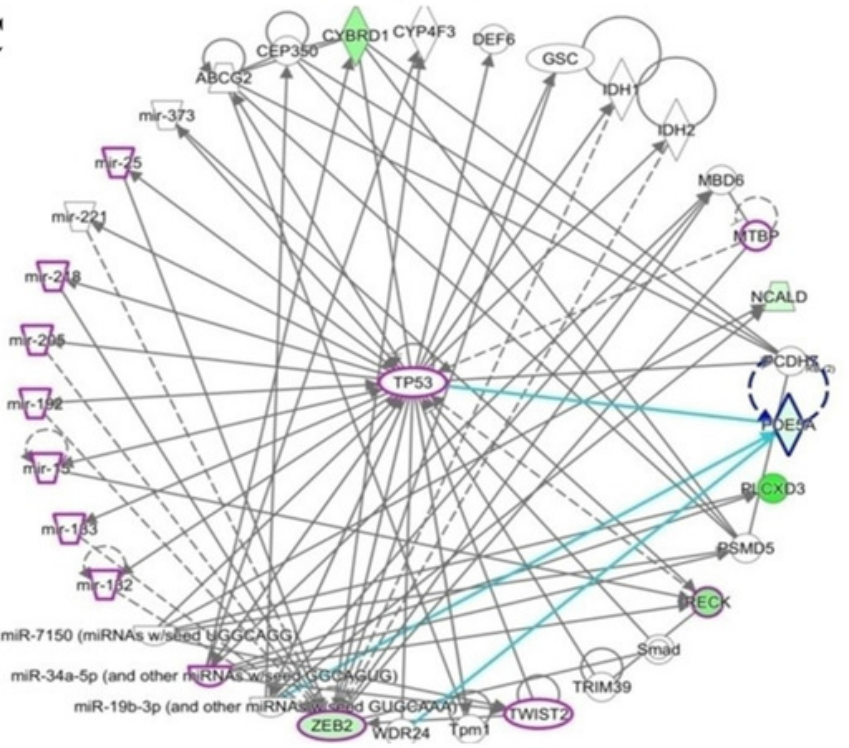

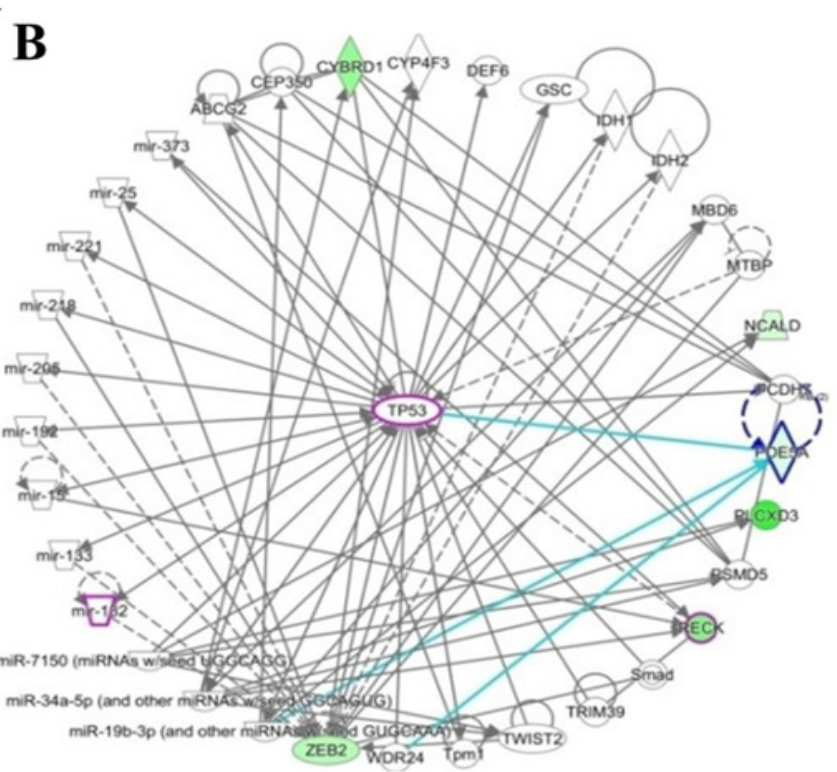

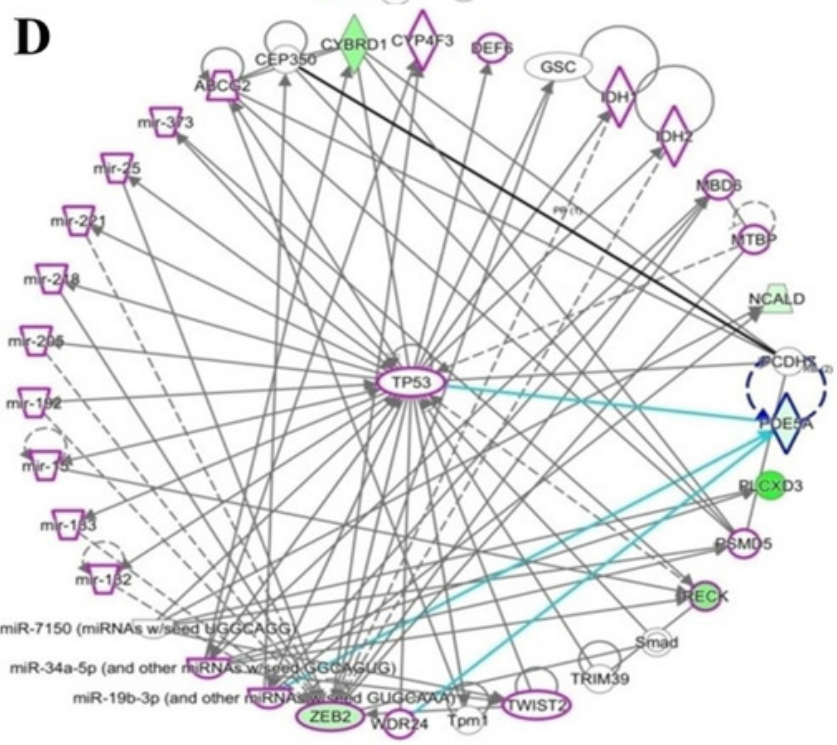


Figure 6

Protein expression analyzed using western blot analysis.

Antibodies against human ZEB2, CYBRD1, PDE5A, and GAPDH were employed as primary antibodies.

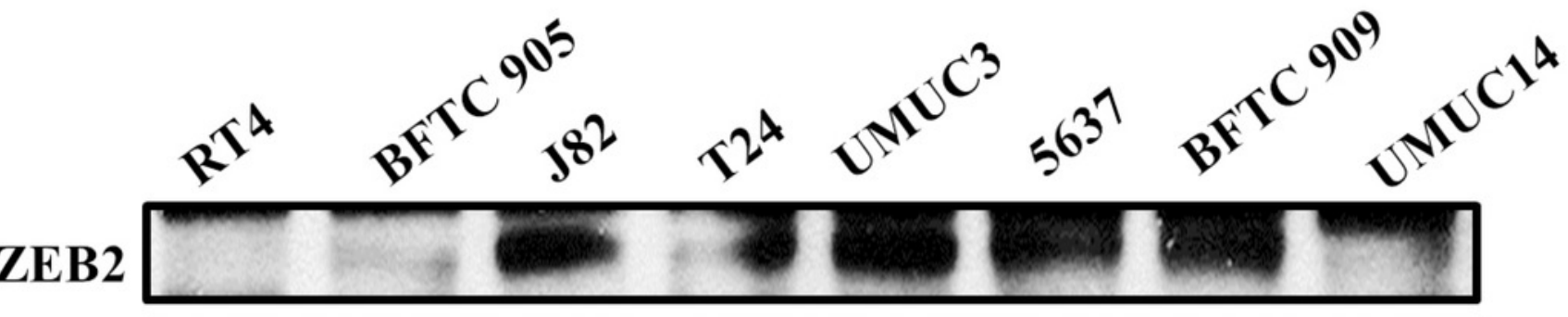

CYBRD1

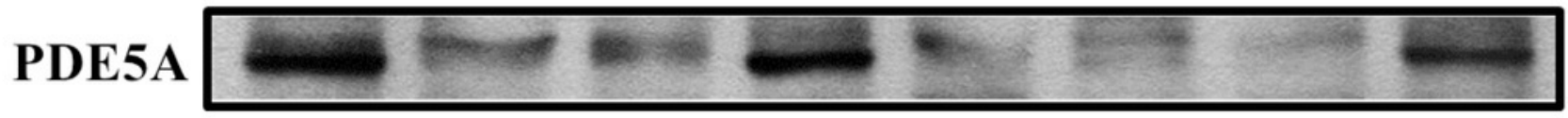

GAPDH $\square$ 


\section{Figure 7}

mRNA expression in UC and UTUC cells

mRNA expression of (A) PDE5A, (B) CYBRD1, and (C) ZEB2 in UC and UTUC cells. J82, T24, 5637, and BFTC 909 cells exhibited higher expression of CYBRD1 and ZEB2 but lower expression of PDE5A than RT4 cells. (D) Expression of fold of control levels.

A

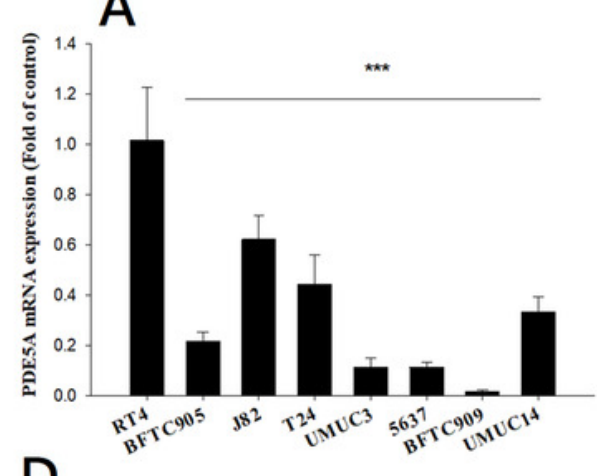

B

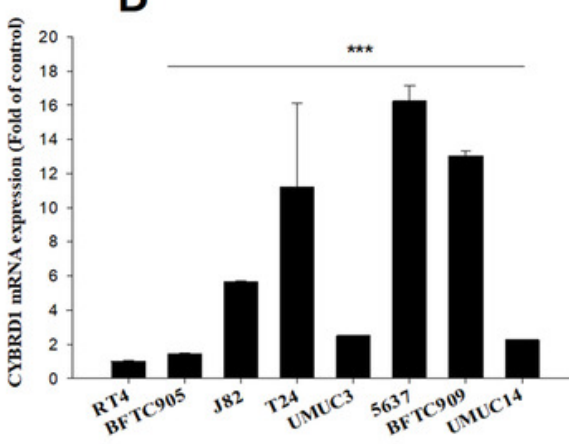

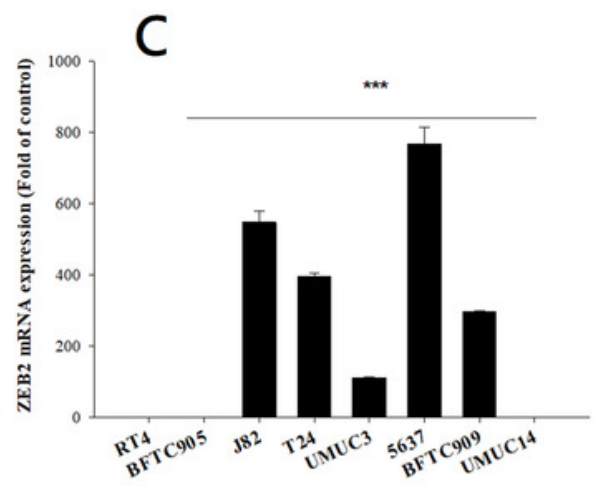

\begin{tabular}{|c|c|c|c|}
\hline qPCR & $\begin{array}{c}\text { PDE5A } \\
\text { (Fold of control) }\end{array}$ & $\begin{array}{c}\text { CYBRD1 } \\
\text { (Fold of control) }\end{array}$ & $\begin{array}{c}\text { ZEB2 } \\
\text { (Fold of control) }\end{array}$ \\
\hline RT4 & $1.00 \pm 0.21$ & $1.00 \pm 0.05$ & $1.00 \pm 0.20$ \\
\hline BFTC905 & $0.22 \pm 0.04 * * *$ & $1.46 \pm 0.04 * * *$ & $0.12 \pm 0.07 * * *$ \\
\hline J82 & $0.62 \pm 0.09^{* * *}$ & $5.65 \pm 0.07 * * *$ & $548.55 \pm 31.12^{* * *}$ \\
\hline T24 & $0.44 \pm 0.12^{* * *}$ & $11.21 \pm 4.90^{* * *}$ & $397.39 \pm 8.65^{* * *}$ \\
\hline UMUC3 & $0.11 \pm 0.03^{* * *}$ & $2.50 \pm 0.03 * * *$ & $111.40 \pm 2.66^{* * *}$ \\
\hline 5637 & $0.11 \pm 0.02^{* * *}$ & $16.24 \pm 0.92^{* * *}$ & $768.38 \pm 46.19^{* * *}$ \\
\hline BFTC909 & $0.02 \pm 0.01^{* * *}$ & $13.02 \pm 0.28^{* * *}$ & $297.04 \pm 2.94 * * *$ \\
\hline UMUC14 & $0.69 \pm 0.11^{* * *}$ & $2.26 \pm 0.01 * * *$ & $0.33 \pm 0.06^{* * *}$ \\
\hline
\end{tabular}


Figure 8

Illustration of histopathological analysis of sections from surgical resected human UTUC tissue

(A). It shows different levels of localization for PDE5A, CYBRD1, and ZEB2 staining in 200X magnification . Scale bar, $100 \mu \mathrm{m}$. (B) Positive control= breast tumor tissue with higher PDE5A expression. (C) Reagent control=without primary antibody. 


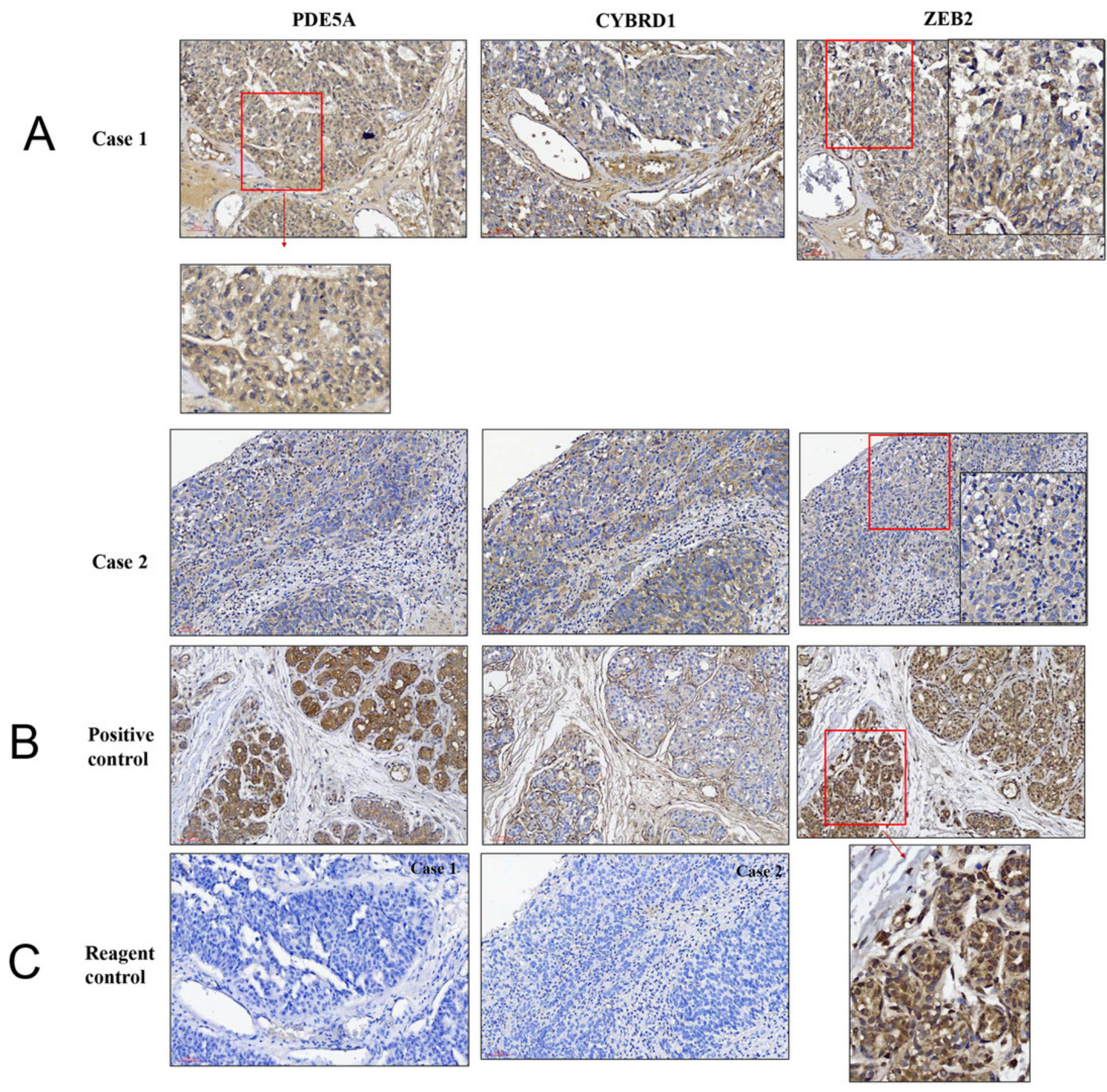




\section{Figure 9}

Paired UTUC tissues (normal and tumor) from 20 patients were evaluated for PDE 5A expression through real-time PCR.

GAPDH was used as an internal control. The relative expression of PDE5 in tumors was significantly lower than that in normal UTUC tissues

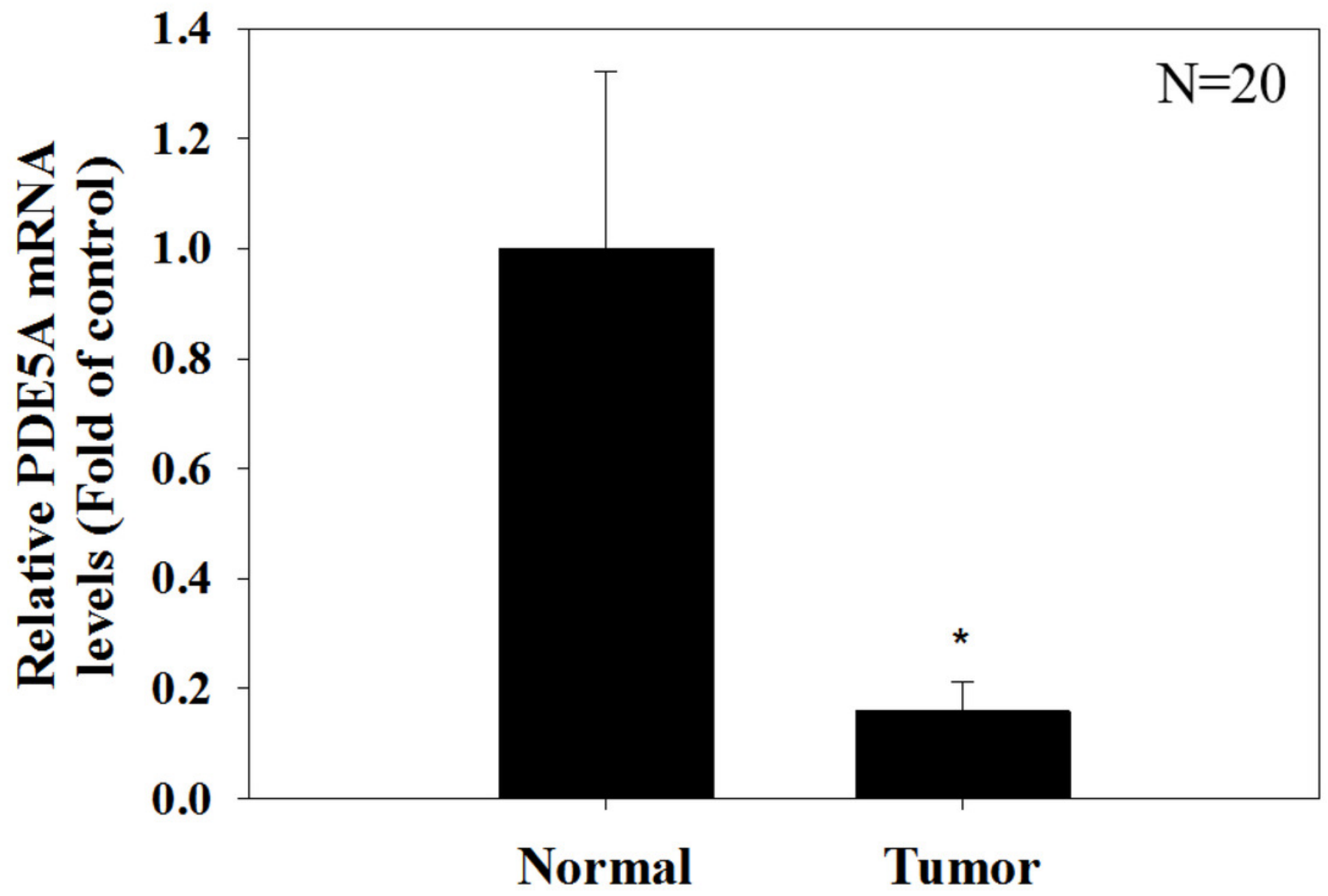




\section{Table $\mathbf{1}$ (on next page)}

The characteristics of two patients whose specimens were for NGS analysis 
1 Table 1 The characteristics of two patients whose specimens were for NGS analysis

\begin{tabular}{lll}
\hline & Patient 1 & Patient 2 \\
\hline Gender & Female & Female \\
Age & 67 & 76 \\
Tumor site & Renal pelvis & Renal pelvis \\
Laterality & Left & Left \\
Tumor grading & high & high \\
Pathology T stage & T3 & T1 \\
$\quad$ N stage & N0 & N0 \\
$\quad$ M stage & M0 & M0 \\
Lymphovascular invasion & No & No \\
Perineural invasion & No & No \\
\hline
\end{tabular}

2

3 


\section{Table 2 (on next page)}

Up expression of miRNA in renal pelvis cancer compared to adjacent normal tissue using next-generation sequencing 
1 Table 2 Up expression of miRNA in renal pelvis cancer compared to adjacent normal tissue

2 using next-generation sequencing

\begin{tabular}{|c|c|c|c|c|}
\hline miRNA name & precursor & $\begin{array}{l}\text { Fold Change } \\
\text { T1/N1 }\end{array}$ & $\begin{array}{l}\text { Fold Change } \\
\text { T2/N2 }\end{array}$ & $\begin{array}{l}\text { Direction } \\
\text { of change }\end{array}$ \\
\hline \multirow[t]{2}{*}{ hsa-miR-128-3p } & hsa-mir-128-2 & 5.393939394 & 2.482288828 & $\mathrm{Up}$ \\
\hline & hsa-mir-128-1 & 5.74426413 & 2.427990236 & Up \\
\hline hsa-miR-200a-5p & hsa-mir-200a & 7.921787709 & 5.284688995 & $\mathrm{Up}$ \\
\hline hsa-miR-200a-3p & hsa-mir-200a & 10.04772448 & 4.169089518 & $\mathrm{Up}$ \\
\hline hsa-miR-200b-3p & hsa-mir-200b & 9.661833489 & 5.984396618 & $\mathrm{Up}$ \\
\hline hsa-miR-200c-3p & hsa-mir-200c & 12.07910337 & 6.287042777 & $\mathrm{Up}$ \\
\hline hsa-miR-181c-3p & hsa-mir-181c & 3.812911726 & 6.021377672 & Up \\
\hline hsa-miR-452-5p & hsa-mir-452 & 5.802035153 & 5.31097561 & Up \\
\hline hsa-miR-210-3p & hsa-mir-210 & 24.96411483 & 6.72866242 & Up \\
\hline hsa-miR-1307-3p & hsa-mir-1307 & 4.069922309 & 6.355513308 & Up \\
\hline hsa-miR-130b-3p & hsa-mir-130b & 9.120198265 & 6.013647643 & Up \\
\hline hsa-miR-149-5p & hsa-mir-149 & 7.352219075 & 5.438751472 & Up \\
\hline hsa-miR-106b-5p & hsa-mir-106b & 6.602572783 & 2.809605489 & Up \\
\hline hsa-miR-421 & hsa-mir-421 & 5.075050033 & 2.636952998 & Up \\
\hline hsa-miR-345-5p & hsa-mir-345 & 4.386983632 & 6.092846271 & Up \\
\hline hsa-miR-454-3p & hsa-mir-454 & 3.7627829 & 2.296692607 & Up \\
\hline hsa-miR-1307-5p & hsa-mir-1307 & 4.343764381 & 3.805475504 & Up \\
\hline hsa-miR-1260b & hsa-mir-1260b & 3.885969522 & 3.145619087 & Up \\
\hline hsa-miR-1260a & hsa-mir-1260a & 3.365115228 & 2.901953557 & Up \\
\hline hsa-miR-181d-5p & hsa-mir-181d & 4.586446105 & 6.408422725 & Up \\
\hline hsa-miR-20a-5p & hsa-mir-20a & 11.61530945 & 6.656237624 & $\mathrm{Up}$ \\
\hline hsa-miR-17-5p & hsa-mir-17 & 10.48332695 & 4.792083013 & Up \\
\hline hsa-miR-425-5p & hsa-mir-425 & 8.732341228 & 7.380424454 & $\mathrm{Up}$ \\
\hline hsa-miR-34a-5p & hsa-mir-34a & 3.420600858 & 2.170505872 & Up \\
\hline hsa-miR-429 & hsa-mir-429 & 7.733315748 & 4.339373602 & $\mathrm{Up}$ \\
\hline hsa-miR-301a-3p & hsa-mir-301a & 3.221881391 & 4.98474609 & Up \\
\hline \multirow[t]{5}{*}{ hsa-miR-941 } & hsa-mir-941-1 & 3.413867323 & 2.183439332 & $\mathrm{Up}$ \\
\hline & hsa-mir-941-2 & 3.413867323 & 2.183439332 & Up \\
\hline & hsa-mir-941-3 & 3.413867323 & 2.183439332 & $\mathrm{Up}$ \\
\hline & hsa-mir-941-4 & 3.413867323 & 2.183439332 & Up \\
\hline & hsa-mir-941-5 & 3.413867323 & 2.183439332 & $\mathrm{Up}$ \\
\hline
\end{tabular}




\begin{tabular}{lllll}
\hline hsa-miR-769-5p & hsa-mir-769 & 2.031387504 & 2.621387596 & $\mathrm{Up}$ \\
hsa-miR-335-3p & hsa-mir-335 & 6.192922017 & 5.23965448 & $\mathrm{Up}$ \\
hsa-miR-222-3p & hsa-mir-222 & 2.235965788 & 5.080788558 & $\mathrm{Up}$ \\
\hline hsa-miR-203a-3p & hsa-mir-203a & 21.84072277 & 5.781700835 & $\mathrm{Up}$ \\
hsa-let-7d-5p & hsa-let-7d & 3.475411219 & 3.417895946 & $\mathrm{Up}$ \\
hsa-miR-181c-5p & hsa-mir-181c & 3.920424636 & 5.225985327 & $\mathrm{Up}$ \\
hsa-miR-93-5p & hsa-mir-93 & 5.664360678 & 3.228353016 & $\mathrm{Up}$ \\
hsa-miR-221-3p & hsa-mir-221 & 2.414195555 & 3.433672238 & $\mathrm{Up}$ \\
\hline hsa-miR-151a-5p & hsa-mir-151a & 4.45419659 & 2.681035029 & $\mathrm{Up}$ \\
hsa-miR-151a-3p & hsa-mir-151a & 4.769749212 & 2.990544659 & $\mathrm{Up}$ \\
\hline hsa-miR-183-5p & hsa-mir-183 & 20.03425235 & 17.04563573 & $\mathrm{Up}$ \\
hsa-miR-205-5p & hsa-mir-205 & 14.92682996 & 5.746883622 & $\mathrm{Up}$ \\
hsa-miR-21-3p & hsa-mir-21 & 11.17629621 & 2.581143517 & $\mathrm{Up}$ \\
hsa-miR-98-5p & hsa-mir-98 & 3.019229747 & 5.633611035 & $\mathrm{Up}$ \\
hsa-let-7b-5p & hsa-let-7b & 2.102740307 & 4.321044032 & $\mathrm{Up}$ \\
hsa-miR-141-3p & hsa-mir-141 & 10.55120146 & 2.056804463 & $\mathrm{Up}$ \\
hsa-miR-182-5p & hsa-mir-182 & 24.16001436 & 12.97609881 & $\mathrm{Up}$ \\
\hline hsa-miR-92a-3p & hsa-mir-92a-2 & 5.629857838 & 2.286613948 & $\mathrm{Up}$ \\
\hline hsa-miR-191-5p & hsa-mir-191 & 3.327191237 & 5.037676123 & $\mathrm{Up}$ \\
\hline hsa-let-7a-5p & hsa-let-7a-3 & 2.074203797 & 3.111619867 & $\mathrm{Up}$ \\
\hline & hsa-let-7a-2 & 2.075618201 & 3.108941695 & \\
\hline & hsa-let-7a-1 & 2.076021712 & 3.108625093 & \\
\hline
\end{tabular}

3

4

5 


\section{Table 3 (on next page)}

Down expression of miRNA in renal pelvis cancer compared to adjacent normal tissue using next-generation sequencing 
1 Table 3 Down expression of miRNA in renal pelvis cancer compared to adjacent normal tissue

2 using next-generation sequencing

\begin{tabular}{lllll}
\hline miRNA name & precursor & $\begin{array}{l}\text { Fold Change } \\
\text { T1/N1 }\end{array}$ & $\begin{array}{l}\text { Fold Change } \\
\text { T2/N2 }\end{array}$ & $\begin{array}{l}\text { Direction } \\
\text { of change }\end{array}$ \\
\hline hsa-miR-99a-5p & hsa-mir-99a & -4.218629767 & -40.88795732 & Down \\
hsa-miR-145-3p & hsa-mir-145 & -4.030566925 & -4.695092025 & Down \\
hsa-miR-125b-5p & hsa-mir-125b-1 & -3.344198175 & -16.626662 & Down \\
hsa-miR-125b-5p & hsa-mir-125b-2 & -3.352884586 & -16.58782609 & Down \\
hsa-miR-145-5p & hsa-mir-145 & -4.520230974 & -5.234484965 & Down \\
hsa-miR-100-5p & hsa-mir-100 & -2.517396655 & -30.5010168 & Down \\
hsa-miR-451a & hsa-mir-451a & -3.800722042 & -20.86300667 & Down \\
hsa-let-7c-5p & hsa-let-7c & -2.028677204 & -11.93390878 & Down \\
hsa-miR-143-3p & hsa-mir-143 & -2.352348002 & -3.043986991 & Down \\
\hline
\end{tabular}

3

4 


\section{Table 4 (on next page)}

Candidate genes with putative upstream miRNA and differentially expressed genes from NGS database 
1 Table 4 Candidate genes with putative upstream miRNA and differentially expressed genes from

2 NGS database

\begin{tabular}{lllll}
\hline Up-regulated & Target down- & Gene name & Fold-change & Fold-change \\
& regulated mRNA & (T1 vs. N1) & (T2 vs. N2) \\
microRNA & & &
\end{tabular}

\begin{tabular}{|c|c|c|c|c|}
\hline hsa-miR-181c-5p & $P D E 5 A$ & phosphodiesterase $5 \mathrm{~A}$ & 3.92 & 5.23 \\
\hline hsa-miR-33b-3p & $P D E 5 A$ & & 10.57 & 5.56 \\
\hline hsa-miR-98-3p & $P D E 5 A$ & & 3.39 & 2.41 \\
\hline hsa-miR-200b-3p & $R E C K$ & $\begin{array}{l}\text { reversion-inducing-cysteine- } \\
\text { rich protein with kazal } \\
\text { motifs }\end{array}$ & 9.66 & 5.98 \\
\hline hsa-miR-200c-3p & $R E C K$ & & 12.08 & 6.29 \\
\hline hsa-miR-34a-5p & $R E C K$ & & 3.42 & 2.17 \\
\hline hsa-miR-429 & $R E C K$ & & 7.73 & 4.34 \\
\hline hsa-miR-141-3p & $Z E B 2$ & $\begin{array}{l}\text { zinc finger E-box binding } \\
\text { homeobox } 2\end{array}$ & 10.55 & 2.06 \\
\hline hsa-miR-200a-3p & $Z E B 2$ & & 10.05 & 4.17 \\
\hline hsa-miR-200b-3p & $Z E B 2$ & & 9.66 & 5.98 \\
\hline hsa-miR-200c-3p & $Z E B 2$ & & 12.08 & 6.29 \\
\hline hsa-miR-429 & $Z E B 2$ & & 7.73 & 4.34 \\
\hline hsa-miR-106a-5p & $C Y B R D 1$ & cytochrome $b$ reductase 1 & 5.28 & 2.65 \\
\hline hsa-miR-106b-5p & $C Y B R D 1$ & & 6.60 & 2.81 \\
\hline hsa-miR-17-5p & $C Y B R D 1$ & & 10.48 & 4.79 \\
\hline hsa-miR-20a-5p & $C Y B R D 1$ & & 11.62 & 6.66 \\
\hline hsa-miR-652-5p & $C Y B R D 1$ & & 3.14 & 2.78 \\
\hline hsa-miR-93-5p & $C Y B R D 1$ & & 5.66 & 3.23 \\
\hline hsa-miR-452-5p & CYBRD1 & & 5.80 & 5.31 \\
\hline
\end{tabular}




\section{Table 5 (on next page)}

The clinical characteristics of 20 patients 
1 Table 5 The clinical characteristics of 20 patients

\begin{tabular}{ll}
\hline & Number \\
\hline Gender & Male: 11 \\
& Female: 9 \\
Age (mean, year) & 65.72 \\
Tumor side & Left: 13 \\
& Right: 7 \\
Tumor location & Ureter: 3 \\
& Renal pelvis: 17 \\
Pathology Grade & Low grade: 2 \\
& High grade: 18 \\
Pathology T stage & T1: 6 \\
& T2: 4 \\
& T3: 9 \\
& T4: 1 \\
\hline
\end{tabular}

2

3 